\title{
The Magic Square and Symmetric Compositions II
}

\begin{abstract}
The construction of Freudenthal's Magic Square, which contains the exceptional simple Lie algebras of types $F_{4}, E_{6}, E_{7}$ and $E_{8}$, in terms of symmetric composition algebras is further developed here. The para-Hurwitz algebras, which form a subclass of the symmetric composition algebras, will be defined, in the split case, in terms of the natural two dimensional module for the simple Lie algebra $\mathfrak{s l}_{2}$. As a consequence, it will be shown how all the Lie algebras in Freudenthal's Magic Square can be constructed, in a unified way, using copies of $\mathfrak{s l}_{2}$ and of its natural module.
\end{abstract}

\section{Introduction}

The exceptional simple Lie algebras in Killing-Cartan's classification are fundamental objects in many branches of mathematics and physics. A lot of different constructions of these objects have been given, many of which involve some nonassociative algebras or triple systems.

In 1966 Tits gave a unified construction of the exceptional simple Lie algebras which uses a couple of ingredients: a unital composition algebra $C$ and a simple Jordan algebra $J$ of degree 3 [31]. At least in the split cases, this is a construction which depends on two unital composition algebras, since the Jordan algebra involved consists of the $3 \times 3$-hermitian matrices over a unital composition algebra. Even though the construction is not symmetric in the two composition algebras that are being used, the outcome (the Magic Square) is indeed symmetric.

2000 Mathematics Subject Classification: Primary: 17B25. Secondary: 17A75

Keywords: Freudenthal Magic Square, symmetric composition algebra, triality, exceptional Lie algebra. 
Over the years, more symmetric constructions have been given, starting with a construction by Vinberg in 1966 [30]. Later on, a quite general construction was given by Allison and Faulkner [2] of Lie algebras out of structurable ones. In the particular case of the tensor product of two unital composition algebras, this construction provides another symmetric construction of Freudenthal's Magic Square. Quite recently, Barton and Sudbery $[4,5]$ (see also Landsberg and Manivel [27, 28]) gave a simple recipe to obtain the Magic Square in terms of two unital composition algebras and their triality Lie algebras which, in perspective, is subsumed in Allison-Faulkner's construction.

Let us recall that a composition algebra is a triple $(S, \cdot, q)$, where $(S, \cdot)$ is a (nonassociative) algebra over a field $F$ with multiplication denoted by $x \cdot y$ for $x, y \in S$, and where $q: S \rightarrow F$ is a regular quadratic form (the norm) such that, for any $x, y \in S$ :

$$
q(x \cdot y)=q(x) q(y)
$$

In what follows, the ground field $F$ will always be assumed to be of characteristic $\neq 2$.

Unital composition algebras (or Hurwitz algebras) form a well-known class of algebras. Any Hurwitz algebra has finite dimension equal to either 1, 2, 4 or 8. The two-dimensional Hurwitz algebras are the quadratic étale algebras over the ground field $F$, the four dimensional ones are the generalized quaternion algebras, and the eight dimensional Hurwitz algebras are called Cayley algebras, and are analogues to the classical algebra of octonions (for a nice survey of the latter, see [3]).

However, as shown in [26], the triality phenomenon is better dealt with by means of the so called symmetric composition algebras, instead of the classical unital composition algebras. This led the author [11] to reinterpret the Barton-Sudbery's construction in terms of two symmetric composition algebras.

A composition algebra $(S, *, q)$ is said to be symmetric if it satisfies

$$
q(x * y, z)=q(x, y * z),
$$

where $q(x, y)=q(x+y)-q(x)-q(y)$ is the polar of $q$. In what follows, any quadratic form and its polar will always be denoted by the same letter. Equation (1.2) is equivalent to

$$
(x * y) * x=x *(y * x)=q(x) y
$$

for any $x, y \in S$. (See [26, Ch. VIII] for the basic facts and notations.) 
The classification of the symmetric composition algebras was obtained in $[14]$ (for characteristic $\neq 3$, see also $[26, \mathrm{Ch}$. VIII]) and in [10] (for characteristic 3).

Given any Hurwitz algebra $C$ with norm $q$, standard involution $x \mapsto \bar{x}=$ $q(x, 1) 1-x$, and multiplication denoted by juxtaposition, the new algebra defined on $C$ but with multiplication

$$
x \bullet y=\bar{x} \bar{y},
$$

is a symmetric composition algebra, called the associated para-Hurwitz algebra. In dimension 1, 2 or 4, any symmetric composition algebra is a para-Hurwitz algebra, with a few exceptions in dimension 2 which are, nevertheless, forms of para-Hurwitz algebras; while in dimension 8, apart from the para-Hurwitz algebras, there is a new family of symmetric composition algebras termed Okubo algebras.

If $(S, *, q)$ is any symmetric composition algebra, consider the corresponding orthogonal Lie algebra

$$
\mathfrak{o}(S, q)=\left\{d \in \operatorname{End}_{F}(S): q(d(x), y)+q(x, d(y))=0 \forall x, y \in S\right\},
$$

and the subalgebra of $\mathfrak{o}(S, q)^{3}$ defined by

$$
\begin{aligned}
& \operatorname{tri}(S, *, q)= \\
& =\left\{\left(d_{0}, d_{1}, d_{2}\right) \in \mathfrak{o}(S, q)^{3}: d_{0}(x * y)=d_{1}(x) * y+x * d_{2}(y) \forall x, y \in S\right\}
\end{aligned}
$$

The map

$$
\theta: \mathfrak{t r i}(S, *, q) \rightarrow \operatorname{tri}(S, *, q), \quad\left(d_{0}, d_{1}, d_{2}\right) \mapsto\left(d_{2}, d_{0}, d_{1}\right),
$$

is an automorphism of $\operatorname{tri}(S, *, q)$ of order 3 , the triality automorphism. Its fixed subalgebra is (isomorphic to) the derivation algebra of the algebra $(S, *)$ which, if the dimension is 8 and the characteristic of the ground field is $\neq 2,3$, is a simple Lie algebra of type $G_{2}$ in the para-Hurwitz case and a simple Lie algebra of type $A_{2}$ (a form of $\mathfrak{s l}_{3}$ ) in the Okubo case.

For any $x, y \in S$, the triple

$$
t_{x, y}=\left(\sigma_{x, y}, \frac{1}{2} q(x, y) i d-r_{x} l_{y}, \frac{1}{2} q(x, y) i d-l_{x} r_{y}\right)
$$

is in $\operatorname{tri}(S, *, q)$, where $\sigma_{x, y}(z)=q(x, z) y-q(y, z) x, r_{x}(z)=z * x$, and $l_{x}(z)=x * z$ for any $x, y, z \in S$.

The construction given in [11] starts with two symmetric composition algebras $(S, *, q)$ and $\left(S^{\prime}, *, q^{\prime}\right)$. Then define $\mathfrak{g}=\mathfrak{g}\left(S, S^{\prime}\right)$ to be the $\mathbb{Z}_{2} \times \mathbb{Z}_{2^{-}}$ graded anticommutative algebra such that $\mathfrak{g}_{(\overline{0}, \overline{0})}=\mathfrak{t r i}(S, *, q) \oplus \mathfrak{t r i}\left(S^{\prime}, *, q^{\prime}\right)$, 
$\mathfrak{g}_{(\overline{1}, \overline{0})}=\mathfrak{g}_{(\overline{0}, \overline{1})}=\mathfrak{g}_{(\overline{1}, \overline{1})}=S \otimes S^{\prime}$. (Unadorned tensor products are considered over the ground field $F$.) For any $a \in S$ and $x \in S^{\prime}$, denote by $\iota_{i}(a \otimes x)$ the element $a \otimes x$ in $\mathfrak{g}_{(\overline{1}, \overline{0})}$ (respectively $\left.\mathfrak{g}_{(\overline{0}, \overline{1})}, \mathfrak{g}_{(\overline{1}, \overline{1})}\right)$ if $i=0$ (respectively, $i=1,2)$. Thus

$$
\mathfrak{g}=\mathfrak{g}\left(S, S^{\prime}\right)=\left(\mathfrak{t r i}(S, *, q) \oplus \mathfrak{t r i}\left(S^{\prime}, *, q^{\prime}\right)\right) \oplus\left(\oplus_{i=0}^{2} \iota_{i}\left(S \otimes S^{\prime}\right)\right) .
$$

The anticommutative multiplication on $\mathfrak{g}$ is defined by means of:

- $\mathfrak{g}_{(\overline{0}, \overline{0})}$ is a Lie subalgebra of $\mathfrak{g}$,

- $\left[\left(d_{0}, d_{1}, d_{2}\right), \iota_{i}(a \otimes x)\right]=\iota_{i}\left(d_{i}(a) \otimes x\right),\left[\left(d_{0}^{\prime}, d_{1}^{\prime}, d_{2}^{\prime}\right), \iota_{i}(a \otimes x)\right]=\iota_{i}(a \otimes$ $\left.d_{i}^{\prime}(x)\right)$, for any $\left(d_{0}, d_{1}, d_{2}\right) \in \operatorname{tri}(S, *, q),\left(d_{0}^{\prime}, d_{1}^{\prime}, d_{2}^{\prime}\right) \in \mathfrak{t r i}\left(S^{\prime}, *, q^{\prime}\right), a \in S$ and $x \in S^{\prime}$.

- $\left[\iota_{i}(a \otimes x), \iota_{i+1}(b \otimes y)\right]=\iota_{i+2}((a * b) \otimes(x * y))$ (indices modulo 3), for any $a, b \in S, x, y \in S^{\prime}$.

- $\left[\iota_{i}(a \otimes x), \iota_{i}(b \otimes y)\right]=q^{\prime}(x, y) \theta^{i}\left(t_{a, b}\right)+q(a, b) \theta^{\prime i}\left(t_{x, y}^{\prime}\right)$, for any $i=0,1,2$, $a, b \in S$ and $x, y \in S^{\prime}$, where $t_{a, b} \in \operatorname{tri}(S, *, q)$ (respectively $t_{x, y}^{\prime} \in$ $\left.\operatorname{tri}\left(S^{\prime}, *, q^{\prime}\right)\right)$ is the element in (1.5) for $a, b \in S$ (resp. $\left.x, y \in S^{\prime}\right)$ and $\theta$ (resp. $\left.\theta^{\prime}\right)$ is the triality automorphism of $\mathfrak{t r i}(S, *, q)\left(\operatorname{resp} . \mathfrak{t r i}\left(S^{\prime}, *, q^{\prime}\right)\right)$.

The main result in [11] asserts that, with this multiplication, $\mathfrak{g}\left(S, S^{\prime}\right)$ is a Lie algebra and, if the characteristic of the ground field is $\neq 2,3$, Freudenthal's Magic Square is recovered (Table 1).

\begin{tabular}{cc|ccccc}
\multicolumn{1}{c}{} & \multicolumn{5}{c}{$\operatorname{dim} S$} \\
\cline { 3 - 6 } $\operatorname{dim} S^{\prime}$ & $A_{1}$ & $A_{2}$ & $C_{3}$ & $F_{4}$ \\
& 1 & $A_{2}$ & $A_{2} \oplus A_{2}$ & $A_{5}$ & $E_{6}$ \\
& 4 & $C_{3}$ & $A_{5}$ & $D_{6}$ & $E_{7}$ \\
& 8 & $F_{4}$ & $E_{6}$ & $E_{7}$ & $E_{8}$
\end{tabular}

Table 1: The Magic Square

In [12] it is proved that if $(S, *, q)$ is an Okubo algebra with an idempotent (this is always the case if the ground field does not admit cubic extensions), then there is a para-Cayley algebra $(\hat{S}, \bullet, q)$ defined on the same vector space $S$ such that $\mathfrak{g}\left(S, S^{\prime}\right)$ is isomorphic to $\mathfrak{g}\left(\hat{S}, S^{\prime}\right)$, for any $S^{\prime}$. 
The purpose of this paper is to delve in the construction $\mathfrak{g}\left(S, S^{\prime}\right)$ given in [11], for two split para-Hurwitz algebras. It turns out that the split paraHurwitz algebras of dimension 4 and 8 (para-quaternion and para-octonion algebras) can be constructed using a very simple ingredient: the two dimensional module for the three dimensional simple split Lie algebra $\mathfrak{s l}_{2}$. This will be shown in Section 2.

As a consequence, all the split Lie algebras in the Magic Square will be constructed in very simple terms using copies of $\mathfrak{s l}_{2}$ and of its natural module. The general form will be

$$
\mathfrak{g}=\oplus_{\sigma \in \mathcal{S}} V(\sigma)
$$

where $\mathcal{S}$ will be a set of subsets of $\{1, \ldots, n\}$, for some $n, V(\emptyset)=\oplus_{i=1}^{n} \mathfrak{s l}_{2}$, a direct sum of copies of $\mathfrak{s l}_{2}$, and for $\emptyset \neq \sigma=\left\{i_{1}, \ldots, i_{r}\right\} \in \mathcal{S}, V(\sigma)=$ $V_{i_{1}} \otimes \cdots \otimes V_{i_{r}}$, with $V_{i}$ the two-dimensional natural module for the $i^{\text {th }}$ copy of $\mathfrak{s l}_{2}$ in $V(\emptyset)$. The Lie bracket will appear in terms of natural contractions. The precise formulas will be given in Section 3. Surprisingly, the real division algebra of octonions will appear in these constructions, but in an unexpected way.

Section 4 will be devoted to show how the models obtained in Section 3 of the exceptional simple split Lie algebras give models too of the exceptional simple split Freudenthal triple systems which, together with simple Jordan algebras, were the basic tools used by Freudenthal to construct the Lie algebras in the Magic Square.

\section{Split para-quaternions and para-octonions}

Let $V$ be a two dimensional vector space over a ground field $F$ (of characteristic $\neq 2$ ), endowed with a nonzero skew-symmetric bilinear form $\langle$.$| .$.$\rangle .$ Consider the symplectic Lie algebra

$$
\mathfrak{s p}(V)=\operatorname{span}\left\{\gamma_{a, b}=\langle a \mid .\rangle b+\langle b \mid .\rangle a: a, b \in V\right\},
$$

which coincides with $\mathfrak{s l}(V)$ (endomorphisms of zero trace). The bilinear form allows $Q=V \otimes V$ to be identified with $\operatorname{End}_{F}(V)$ (the split quaternion algebra over $F$ ) by means of:

$$
\begin{aligned}
V \otimes V & \longrightarrow \operatorname{End}_{F}(V) \\
a \otimes b & \mapsto\langle a \mid \cdot\rangle b: v \mapsto\langle a \mid v\rangle b .
\end{aligned}
$$

Since

$$
\langle a \otimes b(v) \mid w\rangle=\langle a \mid v\rangle\langle b \mid w\rangle=-\langle v \mid b \otimes a(w)\rangle,
$$


it follows that for any $a, b \in V$,

$$
\overline{a \otimes b}=-b \otimes a,
$$

where $f \mapsto \bar{f}$ is the symplectic involution of $\operatorname{End}_{F}(V)$ relative to $\langle$.$\left.| . \right\rangle$ (the standard involution as a quaternion algebra). Also, the norm as a quaternion algebra is given by $q(x)=\operatorname{det} x=\frac{1}{2} \operatorname{trace} x \bar{x}$; so its polar form becomes

$$
\begin{aligned}
q(a \otimes b, c \otimes d) & =\operatorname{trace}((a \otimes b)(\overline{c \otimes d})) \\
& =-\operatorname{trace}((a \otimes b)(d \otimes c)) \\
& =-\langle a \mid c\rangle\langle d \mid b\rangle \\
& =\langle a \mid c\rangle\langle b \mid d\rangle .
\end{aligned}
$$

(The natural symmetric bilinear form on $V \otimes V$ induced from $\langle. \mid\rangle$. )

The split Cayley algebra is obtained by means of the Cayley-Dickson doubling process (see [23, Section 2]): $C=Q \oplus Q$ with multiplication, standard involution and norm given by:

$$
\left\{\begin{array}{l}
(x, y)\left(x^{\prime}, y^{\prime}\right)=\left(x x^{\prime}-\overline{y^{\prime}} y, y^{\prime} x+y \overline{x^{\prime}}\right), \\
\overline{(x, y)}=(\bar{x},-y), \\
q((x, y))=q(x)+q(y),
\end{array}\right.
$$

for any $x, y, x^{\prime}, y^{\prime} \in Q$. Therefore, $C=V \otimes V \oplus V \otimes V$ with

$$
\left\{\begin{array}{l}
(a \otimes b, c \otimes d)\left(a^{\prime} \otimes b^{\prime}, c^{\prime} \otimes d^{\prime}\right) \\
\quad=\left(\left\langle a \mid b^{\prime}\right\rangle a^{\prime} \otimes b-\left\langle d \mid d^{\prime}\right\rangle c \otimes c^{\prime},-\left\langle b \mid c^{\prime}\right\rangle a \otimes d^{\prime}-\left\langle c \mid a^{\prime}\right\rangle b^{\prime} \otimes d\right), \\
\overline{(a \otimes b, c \otimes d)}=-(b \otimes a, c \otimes d), \\
q\left((a \otimes b, c \otimes d),\left(a^{\prime} \otimes b^{\prime}, c^{\prime} \otimes d^{\prime}\right)\right)=\left\langle a \mid a^{\prime}\right\rangle\left\langle b \mid b^{\prime}\right\rangle+\left\langle c \mid c^{\prime}\right\rangle\left\langle d \mid d^{\prime}\right\rangle,
\end{array}\right.
$$

for any $a, b, c, d, a^{\prime}, b^{\prime}, c^{\prime}, d^{\prime} \in V$.

The multiplication $x \bullet y=\bar{x} \bar{y}$ in the associated para-Hurwitz algebra (the split para-octonions) $S_{8}$ takes the form:

$$
\begin{aligned}
(a \otimes b, c & \otimes d) \bullet\left(a^{\prime} \otimes b^{\prime}, c^{\prime} \otimes d^{\prime}\right) \\
& =(b \otimes a, c \otimes d)\left(b^{\prime} \otimes a^{\prime}, c^{\prime} \otimes d^{\prime}\right) \\
& =\left(\left\langle b \mid a^{\prime}\right\rangle b^{\prime} \otimes a-\left\langle d \mid d^{\prime}\right\rangle c \otimes c^{\prime},-\left\langle a \mid c^{\prime}\right\rangle b \otimes d^{\prime}-\left\langle c \mid b^{\prime}\right\rangle a^{\prime} \otimes d\right) .
\end{aligned}
$$

The split para-quaternion algebra $S_{4}$ is just the subalgebra consisting of the first copy of $V \otimes V$ in $S_{8}$. 
The Lie algebra $\mathfrak{s p}(V)^{4}$ acts naturally on $S_{8}$, where the $i^{\text {th }}$ component of $\mathfrak{s p}(V)^{4}$ acts on the $i^{\text {th }}$ copy of $V$ in $S_{8}=V \otimes V \oplus V \otimes V$. This gives an embedding into the orthogonal Lie algebra of $S_{8}$ relative to $q$ :

$$
\rho: \mathfrak{s p}(V)^{4} \longrightarrow \mathfrak{o}\left(S_{8}, q\right) .
$$

Actually, this is an isomorphism of $\mathfrak{s p}(V)^{4}$ onto the subalgebra $\mathfrak{o}(V \otimes V) \oplus$ $\mathfrak{o}(V \otimes V)$ of $\mathfrak{o}\left(S_{8}, q\right)$, which is the even part of $\mathfrak{o}\left(S_{8}, q\right)$ relative to the $\mathbb{Z}_{2^{-}}$ grading given by the orthogonal decomposition $S_{8}=V \otimes V \perp V \otimes V$.

Consider also the linear map (denoted by $\rho$ too):

$$
\rho: V^{\otimes 4} \longrightarrow \mathfrak{o}\left(S_{8}, q\right),
$$

such that

$$
\begin{aligned}
\rho\left(v_{1} \otimes v_{2} \otimes v_{3} \otimes v_{4}\right)\left(\left(w_{1} \otimes w_{2}, w_{3} \otimes w_{4}\right)\right) \\
=\left(\left\langle v_{3} \mid w_{3}\right\rangle\left\langle v_{4} \mid w_{4}\right\rangle v_{1} \otimes v_{2},-\left\langle v_{1} \mid w_{1}\right\rangle\left\langle v_{2} \mid w_{2}\right\rangle v_{3} \otimes v_{4}\right),
\end{aligned}
$$

for any $v_{i}, w_{i} \in V, i=1,2,3,4$. Consider for any $x, y \in S_{8}$ the linear map

$$
\sigma_{x, y}=q(x, .) y-q(y, .) x
$$

(these maps span $\left.\mathfrak{o}\left(S_{8}, q\right)\right)$. Then,

$$
\rho\left(v_{1} \otimes v_{2} \otimes v_{3} \otimes v_{4}\right)=-\sigma_{\left(v_{1} \otimes v_{2}, 0\right),\left(0, v_{3} \otimes v_{4}\right)} .
$$

Observe that $\rho\left(v_{1} \otimes v_{2} \otimes v_{3} \otimes v_{4}\right)$ fills the odd part of the $\mathbb{Z}_{2}$-grading of $\mathfrak{o}\left(S_{8}, q\right)$ mentioned above.

Now, for any $v_{i}, w_{i} \in V, i=1,2,3,4$; a straightforward computation, using that $\langle a \mid b\rangle c+\langle b \mid c\rangle a+\langle c \mid a\rangle b=0$ for any $a, b, c \in V$ (since $\operatorname{dim} V=2$ and the expression is skew symmetric on its arguments), gives:

$$
\begin{aligned}
& {\left[\rho\left(v_{1} \otimes v_{2} \otimes v_{3} \otimes v_{4}\right), \rho\left(w_{1} \otimes w_{2} \otimes w_{3} \otimes w_{4}\right)\right]} \\
& =\frac{1}{2} \sum_{i=1}^{4}\left(\prod_{j \neq i}\left\langle v_{j} \mid w_{j}\right\rangle\right) \nu_{i}\left(\gamma_{v_{i}, w_{i}}\right) \in \rho\left(\mathfrak{s p}(V)^{4}\right),
\end{aligned}
$$

where $\nu_{i}: \mathfrak{s p}(V) \hookrightarrow \mathfrak{s p}(V)^{4}$ denotes the inclusion into the $i^{\text {th }}$ component.

In the same vein, for any $v_{i}, w_{i} \in V, i=1,2,3,4$.

$$
\left\{\begin{array}{l}
\sigma_{\left(v_{1} \otimes v_{2}, 0\right),\left(w_{1} \otimes w_{2}, 0\right)}=\frac{1}{2}\left(\left\langle v_{2} \mid w_{2}\right\rangle \nu_{1}\left(\gamma_{v_{1}, w_{1}}\right)+\left\langle v_{1} \mid w_{1}\right\rangle \nu_{2}\left(\gamma_{v_{2}, w_{2}}\right)\right), \\
\sigma_{\left(0, v_{3} \otimes v_{4}\right),\left(0, w_{3} \otimes w_{4}\right)}=\frac{1}{2}\left(\left\langle v_{4} \mid w_{4}\right\rangle \nu_{3}\left(\gamma_{v_{3}, w_{3}}\right)+\left\langle v_{3} \mid w_{3}\right\rangle \nu_{4}\left(\gamma_{v_{4}, w_{4}}\right)\right) .
\end{array}\right.
$$

The following result (see also $[28,3.4]$ ) summarizes most of the above arguments. 
64 A. Elduque

Proposition 2.9 The vector space

$$
\mathfrak{d}_{4}=\mathfrak{s p}(V)^{4} \oplus V^{\otimes 4},
$$

with anticommutative multiplication given by:

- $\mathfrak{s p}(V)^{4}$ is a Lie subalgebra of $\mathfrak{d}_{4}$,

- for any $s_{i} \in \mathfrak{s p}(V)$ and $v_{i} \in V, i=1,2,3,4$,

$$
\begin{aligned}
& {\left[\left(s_{1}, s_{2}, s_{3}, s_{4}\right), v_{1} \otimes v_{2} \otimes v_{3} \otimes v_{4}\right]} \\
& =s_{1}\left(v_{1}\right) \otimes v_{2} \otimes v_{3} \otimes v_{4}+v_{1} \otimes s_{2}\left(v_{2}\right) \otimes v_{3} \otimes v_{4} \\
& \quad+v_{1} \otimes v_{2} \otimes s_{3}\left(v_{3}\right) \otimes v_{4}+v_{1} \otimes v_{2} \otimes v_{3} \otimes s_{4}\left(v_{4}\right),
\end{aligned}
$$

- for any $v_{i}, w_{i} \in V, i=1,2,3,4$,

$$
\left[v_{1} \otimes v_{2} \otimes v_{3} \otimes v_{4}, w_{1} \otimes w_{2} \otimes w_{3} \otimes w_{4}\right]=\frac{1}{2} \sum_{i=1}^{4}\left(\prod_{j \neq i}\left\langle v_{j} \mid w_{j}\right\rangle\right) \nu_{i}\left(\gamma_{v_{i}, w_{i}}\right),
$$

is a Lie algebra isomorphic to $\mathfrak{o}\left(S_{8}, q\right)$ by means of the linear map $\rho$ defined by (2.3) and (2.4).

Note that $\mathfrak{d}_{4}$ is generated by $V^{\otimes 4}$ and that the decomposition

$$
\mathfrak{d}_{4}=\mathfrak{s p}(V)^{4} \oplus V^{\otimes 4}
$$

is a $\mathbb{Z}_{2}$-grading.

Let us consider now, as in $[28,3.4]$, the order three automorphism $\theta$ : $\mathfrak{d}_{4} \rightarrow \mathfrak{d}_{4}$ such that:

$$
\left\{\begin{array}{l}
\theta\left(\left(s_{1}, s_{2}, s_{3}, s_{4}\right)\right)=\left(s_{3}, s_{1}, s_{2}, s_{4}\right) \\
\theta\left(v_{1} \otimes v_{2} \otimes v_{3} \otimes v_{4}\right)=v_{3} \otimes v_{1} \otimes v_{2} \otimes v_{4}
\end{array}\right.
$$

for any $s_{i} \in \mathfrak{s p}(V)$ and $v_{i} \in v, i=1,2,3,4$. Then:

Proposition 2.12 (Local triality) For any $f \in \mathfrak{d}_{4}$ and any $x, y \in S_{8}$ :

$$
\rho(f)(x \bullet y)=\left(\rho\left(\theta^{-1}(f)\right)(x)\right) \bullet y+x \bullet\left(\rho\left(\theta^{-2}(f)\right)(y)\right) .
$$

Proof. It is enough to prove this for generators of $\mathfrak{d}_{4}$ and of $S_{8}$, and hence for $f=v_{1} \otimes v_{2} \otimes v_{3} \otimes v_{4}, x=\left(a_{1} \otimes a_{2}, a_{3} \otimes a_{4}\right), y=\left(b_{1} \otimes b_{2}, b_{3} \otimes b_{4}\right)$, with $v_{i}, a_{i}, b_{i} \in V, i=1,2,3,4$, and this is a straightforward computation. 
The triality Lie algebra of $\left(S_{8}, \bullet, q\right)$ is

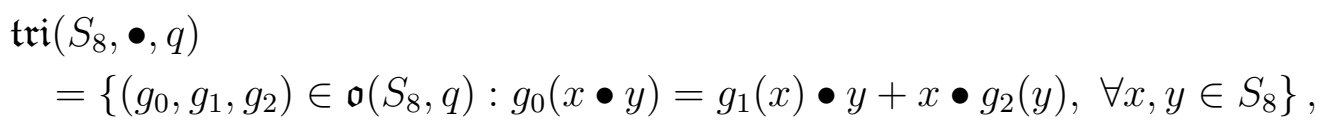

and the projection on any of its components gives an isomorphism among the Lie algebras $\operatorname{tri}\left(S_{8}, \bullet, q\right)$ and $\mathfrak{o}\left(S_{8}, q\right)$. Let $\rho_{i}=\rho \circ \theta^{-i}, i=0,1,2$. By dimension count, the previous Proposition immediately implies:

Corollary 2.13 $\operatorname{tri}\left(S_{8}, \bullet, q\right)=\left\{\left(\rho_{0}(f), \rho_{1}(f), \rho_{2}(f)\right): f \in \mathfrak{d}_{4}\right\}$.

Denote by $V_{i}$ the $\mathfrak{s p}(V)^{4}$-module $V$ on which only the $i^{\text {th }}$ component acts: $\left(s_{1}, s_{2}, s_{3}, s_{4}\right) \cdot v=s_{i}(v)$ for any $s_{i} \in \mathfrak{s p}(V), i=1,2,3,4$, and $v \in V$. Also, denote by $\iota_{i}\left(S_{8}\right)$ the $\mathfrak{d}_{4}$-module associated to the representation $\rho_{i}: \mathfrak{d}_{4} \rightarrow$ $\mathfrak{o}\left(S_{8}, q\right)$. Then, as modules for $\mathfrak{s p}(V)^{4}$ :

$$
\begin{aligned}
& \iota_{0}\left(S_{8}\right)=V_{1} \otimes V_{2} \oplus V_{3} \otimes V_{4}, \\
& \iota_{1}\left(S_{8}\right)=V_{2} \otimes V_{3} \oplus V_{1} \otimes V_{4}, \\
& \iota_{2}\left(S_{8}\right)=V_{3} \otimes V_{1} \oplus V_{2} \otimes V_{4}\left(\simeq V_{1} \otimes V_{3} \oplus V_{2} \otimes V_{4}\right) .
\end{aligned}
$$

Remark 2.15 For the split para-quaternion algebra $S_{4}=V \otimes V$, by restriction we obtain:

$$
\mathfrak{t r i}\left(S_{4}, \bullet, q\right)=\left\{\left(\tilde{\rho}_{0}(f), \tilde{\rho}_{1}(f), \tilde{\rho}_{2}(f)\right): f \in \mathfrak{s p}(V)^{3}\right\},
$$

where $\tilde{\rho}_{i}$ is obtained by restriction of $\rho_{i}$ :

$$
\tilde{\rho}_{i}\left(\left(s_{1}, s_{2}, s_{3}\right)\right)=\left.\rho_{i}\left(\left(s_{1}, s_{2}, s_{3}, 0\right)\right)\right|_{V \otimes V} .
$$

Remark 2.16 The construction of $\mathfrak{d}_{4}$ in Proposition 2.9 makes it clear an action of the symmetric group $S_{3}$ on $\mathfrak{d}_{4}$ leaving fixed the last copy of $\mathfrak{s p}(V)$ and of $V$, as shown by the action of $\theta$ in (2.11). As such, $\mathfrak{d}_{4}$ is the natural example of a Lie algebra with triality, as defined in [22].

Moreover, the fixed subalgebra by $\theta$ in $(2.11)$ is a direct sum $(\mathfrak{s p}(V) \oplus$ $\mathfrak{s p}(V)) \oplus S^{3}(V) \otimes V$, where the first copy of $\mathfrak{s p}(V)$ is the diagonal subalgebra in the direct sum of the first three copies of $\mathfrak{s p}(V)$ in $(2.10), S^{3}(V)$ is the module of symmetric tensors in $V^{\otimes 3}$ (the tensor product of the first three copies of $V$ in (2.10)). If the characteristic is $\neq 2,3$, this is the split simple Lie algebra of type $G_{2}$.

The following notation will be useful in the sequel. For any $n \in \mathbb{N}$ and any subset $\sigma \subseteq\{1,2, \ldots, n\}$ consider the $\mathfrak{s p}(V)^{n}$-modules given by

$$
V(\sigma)= \begin{cases}\mathfrak{s} \mathfrak{p}(V)^{n} & \text { if } \sigma=\emptyset, \\ V_{i_{1}} \otimes \cdots \otimes V_{i_{r}} & \text { if } \sigma=\left\{i_{1}, \ldots, i_{r}\right\}, 1 \leq i_{1}<\cdots<i_{r} \leq n .\end{cases}
$$

As before, $V_{i}$ denotes the module $V$ for the $i^{\text {th }}$ component of $\mathfrak{s p}(V)^{n}$, annihilated by the other $n-1$ components. 
Identify any subset $\sigma \subseteq\{1, \ldots, n\}$ with the element $\left(\sigma_{1}, \ldots, \sigma_{n}\right) \in \mathbb{Z}_{2}^{n}$ such that $\sigma_{i}=1$ if and only if $i \in \sigma$. Then for any $\sigma, \tau \in \mathbb{Z}_{2}^{n}$, consider the natural $\mathfrak{s p}(V)^{n}$-invariant maps

$$
\varphi_{\sigma, \tau}: V(\sigma) \times V(\tau) \longrightarrow V(\sigma+\tau)
$$

defined as follows:

- If $\sigma \neq \tau$ and $\sigma \neq \emptyset \neq \tau$, then $\varphi_{\sigma, \tau}$ is obtained by contraction, by means of $\langle$.$| . \rangle$ in the indices $i$ with $\sigma_{i}=1=\tau_{i}$. Thus, for instance,

$\varphi_{\{1,2,3\},\{1,3,4\}}\left(v_{1} \otimes v_{2} \otimes v_{3}, w_{1} \otimes w_{3} \otimes w_{4}\right)=\left\langle v_{1} \mid w_{1}\right\rangle\left\langle v_{3} \mid w_{3}\right\rangle v_{2} \otimes w_{4}$

for any $v_{1}, w_{1} \in V_{1}, v_{2} \in V_{2}, v_{3}, w_{3} \in V_{3}$ and $w_{4} \in V$.

- $\varphi_{\emptyset, \emptyset}$ is the Lie bracket in $\mathfrak{s p}(V)^{n}$.

- For any $\sigma \neq \emptyset, \varphi_{\emptyset, \sigma}=-\varphi_{\sigma, \emptyset}$ is given by the natural action of $\mathfrak{s p}(V)^{n}$ on $V(\sigma)$. Thus, for instance,

$$
\varphi_{\emptyset,\{1,3\}}\left(\left(s_{1}, \ldots, s_{n}\right), v_{1} \otimes v_{3}\right)=s_{1}\left(v_{1}\right) \otimes v_{3}+v_{1} \otimes s_{3}\left(v_{3}\right),
$$

for any $s_{i} \in \mathfrak{s p}(V), i=1, \ldots, n$, and $v_{1} \in V_{1}, v_{3} \in V$.

- Finally, for any $\sigma \neq \emptyset, \varphi_{\sigma, \sigma}$ is given altering slightly (2.7):

$$
\varphi_{\sigma, \sigma}\left(v_{i_{1}} \otimes \cdots \otimes v_{i_{r}}, w_{i_{1}} \otimes \cdots \otimes w_{i_{r}}\right)=-\frac{1}{2} \sum_{j=1}^{r}\left(\prod_{k \neq j}\left\langle v_{i_{k}} \mid w_{i_{k}}\right\rangle \nu_{i_{j}}\left(\gamma_{v_{i_{j}}, w_{i_{j}}}\right)\right) .
$$

(The minus sign here is useful in getting nicer formulae later on.)

With this notation and for $n=4$,

$$
\mathfrak{d}_{4}=V(\emptyset) \oplus V(\{1,2,3,4\}),
$$

with

$$
\left[x_{\sigma}, y_{\tau}\right]=\epsilon_{\mathfrak{d}_{4}}(\sigma, \tau) \varphi_{\sigma, \tau}\left(x_{\sigma}, y_{\tau}\right)
$$

for any $\sigma, \tau \in\{\emptyset,\{1,2,3,4\}\}$ where, for $\sigma=\{1,2,3,4\}$,

$$
\epsilon_{\mathfrak{d}_{4}}(\emptyset, \emptyset)=\epsilon_{\mathfrak{d}_{4}}(\emptyset, \sigma)=\epsilon_{\mathfrak{d}_{4}}(\sigma, \emptyset)=1 \quad \text { and } \quad \epsilon_{\mathfrak{d}_{4}}(\sigma, \sigma)=-1 \text {. }
$$




\section{Exceptional Lie algebras}

In this section, the construction of the split para-quaternions and paraoctonions given in Section 2, together with the description given there of $\mathfrak{d}_{4}$ and of $\mathfrak{t r i}\left(S_{8}, \bullet, q\right)$, will be used to provide constructions of the exceptional simple Lie algebras, which depend only on copies of $\mathfrak{s p}(V)$ and of $V$. (Notations as in the previous section.)

\section{1. $\mathrm{F}_{4}$}

Let us start with the split Lie algebra of type $F_{4}$. Because of (1.6), (2.14) and (2.10), and identifying $\operatorname{tri}\left(S_{8}, \bullet, q\right)$ with $\mathfrak{d}_{4}$ by means of $\rho_{0}$ (Corollary 2.13), one has:

$$
\mathfrak{f}_{4}=\mathfrak{g}\left(S_{8}, F\right)=\oplus_{\sigma \in \mathcal{S}_{\mathfrak{f}_{4}}} V(\sigma),
$$

where

$$
\mathcal{S}_{\mathfrak{f}_{4}}=\{\emptyset,\{1,2,3,4\},\{1,2\},\{2,3\},\{1,3\},\{3,4\},\{1,4\},\{2,4\}\} \subseteq 2^{\{1,2,3,4\}} .
$$

By $\mathfrak{s p}(V)^{4}$-invariance of the Lie bracket in $\mathfrak{f}_{4}$, it follows that

$$
\left[x_{\sigma}, y_{\tau}\right]=\epsilon_{\mathfrak{f}_{4}}(\sigma, \tau) \varphi_{\sigma, \tau}\left(x_{\sigma}, y_{\tau}\right)
$$

for any $\sigma, \tau \in \mathcal{S}_{\mathfrak{f}_{4}}, x_{\sigma} \in V(\sigma)$ and $y_{\tau} \in V(\tau)$; for a suitable map $\epsilon_{\mathfrak{f}_{4}}$ : $\mathcal{S}_{\mathfrak{f}_{4}} \times \mathcal{S}_{\mathfrak{f}_{4}} \rightarrow F$.

Actually, the product $\bullet$ in $(2.2)$ becomes in $\mathfrak{g}\left(S_{8}, F\right)$ a bilinear map

$$
\iota_{0}\left(S_{8} \otimes F\right) \times \iota_{1}\left(S_{8} \otimes F\right) \rightarrow \iota_{2}\left(S_{8} \otimes F\right)
$$

(and cyclically); that is, a bilinear map

$$
\left(V_{1} \otimes V_{2} \oplus V_{3} \otimes V_{4}\right) \times\left(V_{2} \otimes V_{3} \oplus V_{1} \otimes V_{4}\right) \rightarrow\left(V_{3} \otimes V_{1} \oplus V_{2} \otimes V_{4}\right)
$$

given by

$$
\begin{aligned}
& \left(\left(a_{1} \otimes a_{2}, a_{3} \otimes a_{4}\right),\left(b_{2} \otimes b_{3}, b_{1} \otimes b_{4}\right)\right) \\
& \quad \mapsto\left(\left\langle a_{2} \mid b_{2}\right\rangle b_{3} \otimes a_{1}-\left\langle a_{4} \mid b_{4}\right\rangle a_{3} \otimes b_{1},-\left\langle a_{1} \mid b_{1}\right\rangle a_{2} \otimes b_{4}-\left\langle a_{3} \mid b_{3}\right\rangle b_{1} \otimes a_{4}\right),
\end{aligned}
$$

and cyclically. Thus it consists of maps $\varphi_{\sigma, \tau}$ scaled by \pm 1 . Also, equation (2.5) determines the Lie bracket in $\mathfrak{g}\left(S_{8}, F\right)$ between $V(\{1,2,3,4\})$ and $V(\{1,2\}) \oplus V(\{3,4\})$. By cyclic symmetry using $\rho_{1}$ and $\rho_{2}$ one gets all the brackets between $V(\{1,2,3,4\})$ and the $V(\sigma)$ 's with $\sigma \in \mathcal{S}_{\mathfrak{f}_{4}}$ consisting of two elements. Finally, for any $x, y \in S_{8},\left[\iota_{0}(x \otimes 1), \iota_{0}(y \otimes 1)\right]=2 t_{x, y}(1.5)$, 


\begin{tabular}{|c|c|c|c|c|c|c|c|c|}
\hline$\epsilon_{\mathfrak{f}_{4}}$ & $\emptyset$ & $\{1,2\}$ & $\{2,3\}$ & $\{1,3\}$ & $\{1,2,3,4\}$ & $\{3,4\}$ & $\{1,4\}$ & $\{2,4\}$ \\
\hline$\emptyset$ & 1 & 1 & 1 & 1 & 1 & 1 & 1 & 1 \\
\hline$\{1,2\}$ & 1 & -2 & 1 & 1 & 1 & -2 & -1 & -1 \\
\hline$\{2,3\}$ & 1 & 1 & -2 & 1 & 1 & -1 & -2 & -1 \\
\hline$\{1,3\}$ & 1 & 1 & 1 & -2 & 1 & -1 & -1 & -2 \\
\hline$\{1,2,3,4\}$ & 1 & -1 & -1 & -1 & -1 & 1 & 1 & 1 \\
\hline$\{3,4\}$ & 1 & 2 & -1 & -1 & -1 & -2 & -1 & -1 \\
\hline$\{1,4\}$ & 1 & -1 & 2 & -1 & -1 & -1 & -2 & -1 \\
\hline$\{2,4\}$ & 1 & -1 & -1 & 2 & -1 & -1 & -1 & -2 \\
\hline
\end{tabular}

Table 2: $\epsilon_{\mathfrak{f}_{4}}$

since $q^{\prime}(1,1)=2$ in the symmetric composition algebra $F$. But under $\rho_{0}=\rho$, $t_{x, y}$ corresponds to $\sigma_{x, y} \in \mathfrak{o}\left(S_{8}, q\right)$. Then equations (2.6) and (2.8) determine the corresponding values of $\epsilon_{\mathfrak{f}_{4}}$. By cyclic symmetry, one completes the information about $\epsilon_{\mathfrak{f}_{4}}$, which is displayed on Table 2. That is, $\epsilon_{\mathfrak{f}_{4}}$ takes the following values for $\sigma, \tau \subseteq\{1,2,3,4\}$ :

$$
\epsilon_{\mathfrak{f}_{4}}(\sigma, \tau)=\left\{\begin{aligned}
1 & \text { if } \sigma \text { or } \tau \text { is empty, } \\
-1 & \text { if } \sigma=\tau=\{1,2,3,4\}, \\
-2 & \text { if } \emptyset \neq \sigma=\tau \neq\{1,2,3,4\}, \\
2 & \text { if } 4 \in \sigma=\{1,2,3,4\} \backslash \tau, \tau \neq \emptyset, \\
-2 & \text { if } 4 \in \tau=\{1,2,3,4\} \backslash \sigma, \sigma \neq \emptyset, \\
1 & \text { if } 4 \notin \sigma \cup \tau, \\
-1 & \text { if } 4 \in \sigma \cup \tau, \sigma \neq \emptyset \neq \tau .
\end{aligned}\right.
$$

The split Lie algebra $C_{3}$ is realized as $\mathfrak{c}_{3}=\mathfrak{g}\left(S_{4}, F\right)$, a subalgebra of $\mathfrak{f}_{4}=\mathfrak{g}\left(S_{8}, F\right)$ (see [12, Theorem 2.6]). Thus, by restriction, one gets

$$
\mathfrak{c}_{3}=\mathfrak{s p}(V)^{3} \oplus V_{1} \otimes V_{2} \oplus V_{2} \otimes V_{3} \oplus V_{1} \otimes V_{3}=\oplus_{\sigma \in \mathcal{S}_{\mathfrak{c}_{3}}} V(\sigma),
$$

where

$$
\mathcal{S}_{\mathfrak{c}_{3}}=\{\emptyset,\{1,2\},\{2,3\},\{1,3\}\} \subseteq 2^{\{1,2,3\}} .
$$

and the multiplication is given by

$$
\left[x_{\sigma}, y_{\tau}\right]=\epsilon_{\mathfrak{c}_{3}}(\sigma, \tau) \varphi_{\sigma, \tau}\left(x_{\sigma}, y_{\tau}\right)
$$

for any $\sigma, \tau \in \mathcal{S}_{\mathfrak{c}_{3}}, x_{\sigma} \in V(\sigma)$ and $y_{\tau} \in V(\tau)$; where $\epsilon_{\mathfrak{c}_{3}}: \mathcal{S}_{\mathfrak{c}_{3}} \times \mathcal{S}_{\mathfrak{c}_{3}} \rightarrow F$ is given by the left top corner of Table 2 . That is,

$$
\epsilon_{\mathfrak{c}_{3}}(\sigma, \tau)= \begin{cases}-2 & \text { if } \sigma=\tau \neq \emptyset \\ 1 & \text { otherwise }\end{cases}
$$




\section{2. $\mathrm{E}_{7}$}

The split simple Lie algebra $E_{7}$ is realized as $\mathfrak{e}_{7}=\mathfrak{g}\left(S_{8}, S_{4}\right)$. Here there are 4 copies of $V$ involved in $S_{8}$ and another 3 copies for $S_{4}$ (3.4). The indices $1,2,3,4$ will be used for $S_{8}$ and the indices $5,6,7$ for $S_{4}$. Therefore, with the same arguments as above,

$$
\mathfrak{e}_{7}=\oplus_{\sigma \in \mathcal{S}_{\mathfrak{e}_{7}}} V(\sigma)
$$

where

$$
\begin{aligned}
& \mathcal{S}_{\mathfrak{e}_{7}}=\{\emptyset,\{1,2,5,6\},\{2,3,6,7\},\{1,3,5,7\}, \\
&\{1,2,3,4\},\{3,4,5,6\},\{1,4,6,7\},\{2,4,5,7\}\} \subseteq 2^{\{1,2,3,4,5,6,7\}}
\end{aligned}
$$

and the multiplication is given by

$$
\left[x_{\sigma}, y_{\tau}\right]=\epsilon_{\mathfrak{e}_{7}}(\sigma, \tau) \varphi_{\sigma, \tau}\left(x_{\sigma}, y_{\tau}\right)
$$

for any $\sigma, \tau \in \mathcal{S}_{\mathfrak{k}_{7}}, x_{\sigma} \in V(\sigma)$ and $y_{\tau} \in V(\tau)$; where $\epsilon_{\mathfrak{e}_{7}}: \mathcal{S}_{\mathfrak{k}_{7}} \times \mathcal{S}_{\mathfrak{k}_{7}} \rightarrow F$ is given by Table 3 .

\begin{tabular}{c|c|c|c|c|c|c|c|c|}
$\epsilon_{\mathfrak{e}_{7}}$ & $\emptyset$ & $\{1,2,5,6\}$ & $2,3,6,7\}\{1,3,5,7\}$ & $\{1,2,3,4\}$ & $\{3,4,5,6\}$ & $1,4,6,7\}$ & $\{2,4,5,7\}$ \\
\hline$\emptyset$ & 1 & 1 & 1 & 1 & 1 & 1 & 1 & 1 \\
\hline$\{1,2,5,6\}$ & 1 & -1 & 1 & -1 & 1 & -1 & -1 & 1 \\
\hline$\{2,3,6,7\}$ & 1 & -1 & -1 & 1 & 1 & 1 & -1 & -1 \\
\hline$\{1,3,5,7\}$ & 1 & 1 & -1 & -1 & 1 & -1 & 1 & -1 \\
\hline$\{1,2,3,4\}$ & 1 & -1 & -1 & -1 & -1 & 1 & 1 & 1 \\
\hline$\{3,4,5,6\}$ & 1 & 1 & -1 & 1 & -1 & -1 & -1 & 1 \\
\hline$\{1,4,6,7\}$ & 1 & 1 & 1 & -1 & -1 & 1 & -1 & -1 \\
\hline$\{2,4,5,7\}$ & 1 & -1 & 1 & 1 & -1 & -1 & 1 & -1 \\
\hline
\end{tabular}

Table 3: $\epsilon_{\mathfrak{e}_{7}}$

All the entries that appear in this table are \pm 1 , and these signs coincide with the signs that appear in the multiplication table of the real algebra of octonions $\mathbb{O}$ : The usual basis of this algebra is $\{1, i, j, k(=i j), l, i l, j l, k l\}$, with multiplication table given in Table 4.

Actually, $\mathfrak{e}_{7}=\mathfrak{g}\left(S_{8}, S_{4}\right)$ is $\mathbb{Z}_{2} \times \mathbb{Z}_{2}$-graded, and $S_{8}$ is naturally $\mathbb{Z}_{2}$-graded (with even part $S_{4}(2.2)$ ). Thus $\mathfrak{e}_{7}$ is $\mathbb{Z}_{2} \times \mathbb{Z}_{2} \times \mathbb{Z}_{2}$-graded. In fact, the decomposition (3.6) is a 'fake' $\mathbb{Z}_{2}^{7}$-grading, as the $\mathbb{Z}_{2}$-linear map

$$
\begin{aligned}
\chi_{3}: \mathbb{Z}_{2}^{3} & \longrightarrow \mathbb{Z}_{2}^{7} \\
\left(x_{1}, x_{2}, x_{3}\right) & \mapsto\left(x_{1}+x_{3}, x_{1}+x_{2}+x_{3}, x_{2}+x_{3}, x_{3}, x_{1}, x_{1}+x_{2}, x_{2}\right)
\end{aligned}
$$

provides a bijection between $\mathbb{Z}_{2}^{3}$ and $\mathcal{S}_{\mathfrak{e}_{7}} \subseteq \mathbb{Z}_{2}^{7}$. Through $\chi_{3}$, we get the $\mathbb{Z}_{2}^{3}$-grading of $\mathfrak{e}_{7}$. 


\begin{tabular}{c|c|c|c|c|c|c|c|c|} 
& 1 & $i$ & $j$ & $k$ & $l$ & $i l$ & $j l$ & $k l$ \\
\hline 1 & 1 & $i$ & $j$ & $k$ & $l$ & $i l$ & $j l$ & $k l$ \\
\hline$i$ & $i$ & -1 & $k$ & $-j$ & $i l$ & $-l$ & $-k l$ & $j l$ \\
\hline$j$ & $j$ & $-k$ & -1 & $i$ & $j l$ & $k l$ & $-l$ & $-i l$ \\
\hline$k$ & $k$ & $j$ & $-i$ & -1 & $k l$ & $-j l$ & $i l$ & $-l$ \\
\hline$l$ & $l$ & $-i l$ & $-j l$ & $-k l$ & -1 & $i$ & $j$ & $k$ \\
\hline$i l$ & $i l$ & $l$ & $-k l$ & $j l$ & $-i$ & -1 & $-k$ & $k$ \\
\hline$j l$ & $j l$ & $k l$ & $l$ & $-i l$ & $-j$ & $k$ & -1 & $-i$ \\
\hline$k l$ & $k l$ & $-j l$ & $i l$ & $l$ & $-k$ & $-j$ & $i$ & -1 \\
\hline
\end{tabular}

Table 4: The Octonions

Following [1], $\mathbb{O}$ is a twisted group algebra $\mathbb{O}=\mathbb{R}_{\phi}\left[\mathbb{Z}_{2}^{3}\right]$. This is the algebra defined on the group algebra $\mathbb{R}\left[\mathbb{Z}_{2}^{3}\right]$ (with basis $\left\{e^{x}: x \in \mathbb{Z}_{2}^{3}\right\}$ and multiplication determined by $\left.e^{x} e^{y}=e^{x+y}\right)$, but with a new multiplication of the basic elements: $e^{x} \cdot e^{y}=\phi(x, y) e^{x+y}$ for any $x, y \in \mathbb{Z}_{2}^{3}$, where $\phi(x, y)=$ $(-1)^{f(x, y)}$ and $f: \mathbb{Z}_{2}^{3} \times \mathbb{Z}_{2}^{3} \rightarrow \mathbb{Z}_{2}$ is given by

$$
f(x, y)=\left(\sum_{i \geq j} x_{i} y_{j}\right)+x_{1} y_{2} y_{3}+x_{2} y_{1} y_{3}+x_{3} y_{1} y_{2},
$$

for $x=\left(x_{1}, x_{2}, x_{3}\right), y=\left(y_{1}, y_{2}, y_{3}\right)$ in $\mathbb{Z}_{2}^{3}$. (There is a discrepancy here with respect to [1] due to the fact that these authors work with the basis $\{1, i, j, k, l, l i, l j, l k\}$.) One can check easily that identifying $i$ with $(1,0,0)$, $j$ with $(0,1,0)$ and $k$ with $(0,0,1)$ one recovers Table 4 .

Note that through $\chi_{3}$, this identifies $i$ with $\{1,2,5,6\}, j$ with $\{2,3,6,7\}$ and $k$ with $\{1,2,3,4\}$. Moreover $\mathcal{S}_{\mathfrak{e}_{7}}=\chi_{3}\left(\mathbb{Z}_{2}^{3}\right)$ and

$$
\epsilon_{\mathfrak{e}_{7}}(\sigma, \tau)=(-1)^{f\left(\chi_{3}^{-1}(\sigma), \chi_{3}^{-1}(\tau)\right)}
$$

for any $\sigma, \tau \in \mathcal{S}_{\mathfrak{k}_{7}}$, thus providing a closed formula for $\epsilon_{\mathfrak{e}_{7}}$.

Also, the split Lie algebra of type $D_{6}$ is realized as $\mathfrak{d}_{6}=\mathfrak{g}\left(S_{4}, S_{4}\right)$, a subalgebra of $\mathfrak{e}_{7}=\mathfrak{g}\left(S_{8}, S_{4}\right)$. Thus, by restriction, one gets

$$
\mathfrak{d}_{6}=\oplus_{\sigma \in \mathcal{S}_{\mathfrak{v}_{6}}} V(\sigma),
$$

where

$$
\mathcal{S}_{\mathfrak{D}_{6}}=\{\emptyset,\{1,2,5,6\},\{2,3,6,7\},\{1,3,5,7\}\} \subseteq 2^{\{1,2,3,5,6,7\}} .
$$

and the multiplication is given by

$$
\left[x_{\sigma}, y_{\tau}\right]=\epsilon_{\mathfrak{d}_{6}}(\sigma, \tau) \chi_{\sigma, \tau}\left(x_{\sigma}, y_{\tau}\right),
$$

for any $\sigma, \tau \in \mathcal{S}_{\mathfrak{d}_{6}}, x_{\sigma} \in V(\sigma)$ and $y_{\tau} \in V(\tau)$; where $\epsilon_{\mathfrak{d}_{6}}: \mathcal{S}_{\mathfrak{d}_{6}} \times \mathcal{S}_{\mathfrak{d}_{6}} \rightarrow F$ is given by the left top corner of Table 3 . 
Therefore, we may consider the $\mathbb{Z}_{2}$-linear map:

$$
\begin{aligned}
\chi_{2}: \mathbb{Z}_{2}^{2} & \longrightarrow \mathbb{Z}_{2}^{6}\left(\cong 2^{\{1,2,3,5,6,7\}}\right) \\
\left(x_{1}, x_{2}\right) & \mapsto\left(x_{1}, x_{1}+x_{2}, x_{2}, x_{1}, x_{1}+x_{2}, x_{2}\right)
\end{aligned}
$$

and the map $f^{\prime}: \mathbb{Z}_{2}^{2} \times \mathbb{Z}_{2}^{2} \rightarrow \mathbb{Z}_{2}$ given by

$$
f^{\prime}\left(\left(x_{1}, x_{2}\right),\left(y_{1}, y_{2}\right)\right)=x_{1} y_{1}+x_{2}\left(y_{1}+y_{2}\right) .
$$

Then, $\mathcal{S}_{\mathfrak{d}_{6}}=\chi_{2}\left(\mathbb{Z}_{2}^{2}\right)$ and

$$
\epsilon_{\mathfrak{d}_{6}}(\sigma, \tau)=(-1)^{f^{\prime}\left(\chi_{2}^{-1}(\sigma), \chi_{2}^{-1}(\tau)\right)}
$$

for any $\sigma, \tau \in \mathcal{S}_{\mathfrak{d}_{6}}$, thus providing a closed formula too for $\epsilon_{\mathfrak{D}_{6}}$.

\section{3. $\mathrm{E}_{8}$}

All the arguments for $E_{7}$ can be easily extended to $E_{8}$. The split simple Lie algebra $E_{8}$ is realized as $\mathfrak{e}_{8}=\mathfrak{g}\left(S_{8}, S_{8}\right)$. Here there are 4 copies of $V$ involved in the first $S_{8}$ and another 4 copies for the second $S_{8}$. The indices $1,2,3,4$ will be used for the first $S_{8}$ and the indices $5,6,7,8$ for the second $S_{8}$. Therefore, with the same arguments as above,

$$
\mathfrak{e}_{8}=\oplus_{\sigma \in \mathcal{S}_{\mathfrak{e}_{8}}} V(\sigma),
$$

where

$$
\begin{aligned}
\mathcal{S}_{\mathfrak{e}_{8}}=\{ & \emptyset,\{1,2,5,6\},\{2,3,6,7\},\{1,3,5,7\}, \\
& \{1,2,3,4\},\{3,4,5,6\},\{1,4,6,7\},\{2,4,5,7\}, \\
& \{3,4,7,8\},\{1,4,5,8\},\{2,4,6,8\},\{5,6,7,8\}, \\
& \{1,2,7,8\},\{2,3,5,8\},\{1,3,6,8\}\} \\
\subseteq & 2^{\{1,2,3,4,5,6,7,8\}} .
\end{aligned}
$$

and the multiplication is given by

$$
\left[x_{\sigma}, y_{\tau}\right]=\epsilon_{\mathfrak{e}_{8}}(\sigma, \tau) \varphi_{\sigma, \tau}\left(x_{\sigma}, y_{\tau}\right)
$$

for any $\sigma, \tau \in \mathcal{S}_{\mathfrak{e}_{8}}, x_{\sigma} \in V(\sigma)$ and $y_{\tau} \in V(\tau)$; where $\epsilon_{\mathfrak{e}_{8}}: \mathcal{S}_{\mathfrak{e}_{8}} \times \mathcal{S}_{\mathfrak{e}_{8}} \rightarrow F$ is given by Table 5 .

The \pm signs that appear in Table 5 are the same as those that appear in the corresponding entry of the multiplication table of $\mathbb{O} \oplus \mathbb{O}=\mathbb{O} \otimes_{\mathbb{R}} \mathbb{R}[\varepsilon]$ (with $\mathbb{R}[\varepsilon]=\mathbb{R} 1 \oplus \mathbb{R} \varepsilon$ and $\varepsilon^{2}=1$ ) in the basis

$$
\begin{aligned}
& \{1 \otimes 1, i \otimes 1, j \otimes 1, k \otimes 1, l \otimes 1,(i l) \otimes 1,(j l) \otimes 1,(k l) \otimes 1, \\
& 1 \otimes \varepsilon, i \otimes \varepsilon, j \otimes \varepsilon, k \otimes \varepsilon,-l \otimes \varepsilon,-(i l) \otimes \varepsilon,-(j l) \otimes \varepsilon,-(k l) \otimes \varepsilon\}
\end{aligned}
$$

(Note the minus signs of the last four elements.) 


\begin{tabular}{|c|c|c|c|c|c|c|c|c|c|c|c|c|c|c|c|}
\hline$\epsilon_{\mathfrak{e}_{8}}$ & $\emptyset$ & $\left\{\begin{array}{l}1 \\
2 \\
5 \\
6\end{array}\right\}$ & $\left\{\begin{array}{l}2 \\
3 \\
6 \\
7\end{array}\right\}$ & $\left\{\begin{array}{l}1 \\
3 \\
5 \\
7\end{array}\right\}$ & $\left\{\begin{array}{l}1 \\
2 \\
3 \\
4\end{array}\right\}$ & $\left\{\begin{array}{l}3 \\
4 \\
5 \\
6\end{array}\right\}$ & $\left\{\begin{array}{l}1 \\
4 \\
6 \\
7\end{array}\right\}$ & $\left\{\begin{array}{l}2 \\
4 \\
5 \\
7\end{array}\right\}$ & $\left\{\begin{array}{l}3 \\
4 \\
7 \\
8\end{array}\right\}$ & $\left\{\begin{array}{l}1 \\
4 \\
5 \\
8\end{array}\right\}$ & $\left\{\begin{array}{l}2 \\
4 \\
6 \\
8\end{array}\right\}$ & $\left\{\begin{array}{l}5 \\
6 \\
7 \\
8\end{array}\right\}$ & $\left\{\begin{array}{l}1 \\
2 \\
7 \\
8\end{array}\right\}$ & $\left\{\begin{array}{l}2 \\
3 \\
5 \\
8\end{array}\right\}$ & $\left.\begin{array}{l}1 \\
3 \\
6 \\
8\end{array}\right\}$ \\
\hline$\emptyset$ & 1 & 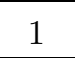 & 1 & $t$ & 1 & 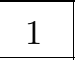 & 1 & 1 & 1 & 1 & 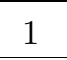 & 1 & 1 & 1 & 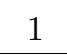 \\
\hline $2,5,6\}$ & 1 & -1 & 1 & -1 & 1 & -1 & -1 & 1 & & 1 & -1 & 1 & -1 & -1 & 1 \\
\hline$, 3,6,7\}$ & 1 & -1 & -1 & 1 & 1 & 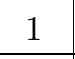 & -1 & -1 & -1 & & 1 & 1 & 1 & -1 & -1 \\
\hline$\{1,3,5,7\}$ & 1 & 1 & -1 & -1 & 1 & -1 & 1 & -1 & 1 & -1 & & 1 & -1 & 1 & -1 \\
\hline$\{1,2,3,4\}$ & 1 & -1 & -1 & -1 & -1 & 1 & 1 & 1 & 1 & 1 & - & & -1 & -1 & -1 \\
\hline$\{3,4,5,6\}$ & 1 & 1 & -1 & 1 & -1 & -1 & -1 & 1 & -1 & 1 & -1 & 1 & & 1 & -1 \\
\hline$\{1,4,6,7\}$ & 1 & 1 & 1 & -1 & -1 & 1 & -1 & -1 & -1 & -1 & 1 & 1 & -1 & & 1 \\
\hline$\{2,4,5,7\}$ & 1 & -1 & 1 & 1 & -1 & -1 & 1 & -1 & 1 & -1 & -1 & 1 & 1 & -1 & \\
\hline $4,7,8\}$ & 1 & & 1 & -1 & -1 & 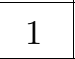 & 1 & -1 & -1 & 1 & -1 & -1 & 1 & 1 & -1 \\
\hline$\{1,4,5,8\}$ & 1 & -1 & & 1 & -1 & -1 & 1 & 1 & -1 & -1 & 1 & -1 & -1 & 1 & 1 \\
\hline$\{2,4,6,8\}$ & 1 & 1 & -1 & & -1 & 1 & -1 & 1 & 1 & -1 & -1 & -1 & 1 & -1 & 1 \\
\hline$\{5,6,7,8\}$ & 1 & -1 & -1 & -1 & & -1 & -1 & -1 & 1 & 1 & 1 & -1 & 1 & 1 & 1 \\
\hline$\{1,2,7,8\}$ & 1 & 1 & -1 & 1 & 1 & & 1 & -1 & -1 & 1 & -1 & -1 & -1 & -1 & 1 \\
\hline$\{2,3,5,8\}$ & 1 & 1 & 1 & -1 & 1 & -1 & & 1 & -1 & -1 & 1 & -1 & 1 & -1 & -1 \\
\hline$\{1,3,6,8\}$ & 1 & -1 & 1 & 1 & 1 & 1 & -1 & & 1 & -1 & -1 & -1 & -1 & 1 & -1 \\
\hline
\end{tabular}

Table 5: $\epsilon_{\mathfrak{e} 8}$

Working with this basis, $\mathbb{O} \otimes_{\mathbb{R}} \mathbb{R}[\varepsilon]$ is shown to be isomorphic to the twisted group algebra $\mathbb{R}_{\Phi}\left[\mathbb{Z}_{2}^{4}\right]$, with $\Phi(x, y)=(-1)^{g(x, y)}$ for $x, y \in \mathbb{Z}_{2}^{4}$, where

$$
\begin{aligned}
g\left(\left(x_{1}, x_{2}, x_{3}, x_{4}\right),\left(y_{1}, y_{2}, y_{3}, y_{4}\right)\right) & \\
& =f\left(\left(x_{1}, x_{2}, x_{3}\right),\left(y_{1}, y_{2}, y_{3}\right)\right)+x_{3} y_{4}+x_{4} y_{3} \\
& =\left(\sum_{1 \leq j \leq i \leq 3} x_{i} y_{j}\right)+x_{3} y_{4}+x_{4} y_{3}+x_{1} y_{2} y_{3}+x_{2} y_{1} y_{3}+x_{3} y_{1} y_{2},
\end{aligned}
$$

with $f$ as in (3.9). The isomorphism carries $i \otimes 1$ to $(1,0,0,0), j \otimes 1$ to $(0,1,0,0), l \otimes 1$ to $(0,0,1,0)$ and $1 \otimes \varepsilon$ to $(0,0,0,1)$; so that, for instance, $l \otimes \varepsilon$ goes to

$$
(-1)^{g((0,0,1,0),(0,0,0,1))}(0,0,1,1)=-(0,0,1,1) .
$$

As for $\mathfrak{e}_{7}$, consider the $\mathbb{Z}_{2}$-linear map

$$
\begin{aligned}
\chi_{4}: \mathbb{Z}_{2}^{4} & \longrightarrow \mathbb{Z}_{2}^{8} \\
\left(x_{1}, x_{2}, x_{3}, x_{4}\right) & \mapsto\left(x_{1}+x_{3}+x_{4}, x_{1}+x_{2}+x_{3}+x_{4}, x_{2}+x_{3}+x_{4},\right. \\
& \left.x_{3}+x_{4}, x_{1}+x_{4}, x_{1}+x_{2}+x_{4}, x_{2}+x_{4}, x_{4}\right)
\end{aligned}
$$


which satisfies that $\mathcal{S}_{\mathfrak{e}_{8}}=\chi_{4}\left(\mathbb{Z}_{2}^{4} \backslash\{(0,0,0,1)\}\right)$ and

$$
\epsilon_{\mathfrak{e}_{8}}(\sigma, \tau)=(-1)^{g\left(\chi_{4}^{-1}(\sigma), \chi_{4}^{-1}(\tau)\right)}
$$

for any $\sigma, \tau \in \mathcal{S}_{\mathfrak{e}_{8}}$, which gives a closed formula for $\epsilon_{\mathfrak{e} 8}$.

Remark 3.19 The models thus constructed for the split $E_{7}$ and $E_{8}$ are strongly related to a very interesting combinatorial construction previously given by A. Grishkov in [21]. To see this, take a basis $\{v, w\}$ of $V$ with $\langle v \mid w\rangle=1$ and denote by $\left\{v_{i}, w_{i}\right\}$ the copy of this basis in $V_{i}$. Take, for instance, $\sigma=\{1,2,7,8\}$ and $\tau=\{1,7,8\} \subseteq \sigma$; then the element $(\sigma, \tau)$ in Grishkov's construction correspond to the element $v_{1} \otimes w_{2} \otimes v_{7} \otimes v_{8}$ in our $V(\sigma) \subseteq \mathfrak{e}_{8}$. It is hoped that this will make Grishkov's construction to appear more natural. In [21] one has to choose an identification of the Moufang loop $M_{7}$ consisting of the elements $\{ \pm 1, \pm i, \pm j \pm k, \pm l, \pm i l, \pm j l, \pm k l\}$ in $\mathbb{O}$ with $\pm \mathcal{S}_{\mathfrak{e}_{7}}$ and another of the Moufang loop $M_{8}=M_{7} \times \mathbb{Z}_{2}$ with $\pm\left(\mathcal{S}_{\mathfrak{e}_{8}} \cup\{\{1,2,3,4,5,6,7,8\}\}\right)$, but no hint is given as how to perform this subtle point. Here such identifications have been explicitly given.

Remark 3.20 The referee has kindly suggested nice interpretations of $\mathcal{S}_{\mathfrak{e}_{7}} \backslash$ $\{\emptyset\}$ as the set of quadrilaterals of the Fano projective plane $\mathbb{Z}_{2} \mathbb{P}^{2}$, and of $\mathcal{S}_{\mathfrak{e}_{8}} \backslash\{\emptyset\}$ as the set of affine planes in the affine space $\mathbb{Z}_{2}^{3}$, once the set $\{1,2,3,4,5,6,7\}$ is suitably identified with the set of points of the Fano plane, and $\{1,2,3,4,5,6,7,8\}$ with $\mathbb{Z}_{2}^{3}$.

For instance, identify the set $\{1,2,3,4,5,6,7\}$ with the set of points of the Fano plane (i.e., the nonzero elements in $\mathbb{Z}_{2}^{3}$ ) as follows:

$$
\begin{aligned}
& 1 \leftrightarrow(1,0,1) \quad 2 \leftrightarrow(1,1,1) \quad 3 \leftrightarrow(0,1,1) \quad 4 \leftrightarrow(0,0,1) \\
& 5 \leftrightarrow(1,0,0) \quad 6 \leftrightarrow(1,1,0) \quad 7 \leftrightarrow(0,1,0)
\end{aligned}
$$

Then the elements of $\mathcal{S}_{\mathfrak{e}_{7}} \backslash\{\emptyset\}$ are precisely the sets of vertices of the quadrilaterals (the complements of the lines) in the Fano plane. By projective duality, these quadrilateral (or better, their complementary lines) are in bijection with the set of points of the Fano plane, and this leads to the bijection of $\mathcal{S}_{\mathfrak{e}_{7}} \backslash\{\emptyset\}$ and $\mathbb{Z}_{2}^{3} \backslash\{(0,0,0)\}$ given by $\chi_{3}$ in (3.8).

Also, the set $\{1,2,3,4,5,6,7,8\}$ may be identified with $\mathbb{Z}_{2}^{3}$ by means of (3.21), together with $8 \leftrightarrow(0,0,0)$. If $l$ is a line in $\mathbb{Z}_{2} \mathbb{P}^{2} \simeq \mathbb{Z}_{2}^{3} \backslash\{\emptyset\}$, then $l \cup\{(0,0,0)\}$ is an affine plane in $\mathbb{Z}_{2}^{3}$ through the origin. On the other hand, if $q$ is a quadrilateral in $\mathbb{Z}_{2} \mathbb{P}^{2}$, then $q$ is an affine plane in $\mathbb{Z}_{2}^{3}$ which does not contain the origin. The elements in $\mathcal{S}_{\mathfrak{e}_{8}} \backslash\{\emptyset\}$ are precisely the affine planes in $\mathbb{Z}_{2}^{3}$, and with our identification (3.21), for any $\left(a_{1}, a_{2}, a_{3}, a_{4}\right) \in \mathbb{Z}_{2}^{4}$, $\chi_{4}\left(a_{1}, a_{2}, a_{3}, a_{4}\right)$, considered as a subset of $\{1,2,3,4,5,6,7,8\}$, and hence of $\mathbb{Z}_{2}^{3}$, is precisely the affine plane with equation $a_{1} x+a_{2} y+a_{3} z+a_{4}=1$. 


\section{4. $\mathrm{E}_{6}$}

For $E_{6}$ things are a bit more involved, partly because it does not contain a subalgebra isomorphic to $\mathfrak{s p}(V)^{6}$. Let $K=F \times F$ be the split two dimensional unital composition algebra and let $S_{2}$ be the associated para-Hurwitz algebra. Thus $S_{2}$ has a basis $\left\{e^{+}, e^{-}\right\}$with multiplication given by

$$
e^{ \pm} \bullet e^{ \pm}=e^{\mp}, \quad e^{ \pm} \bullet e^{\mp}=0,
$$

and with norm given by $q\left(e^{ \pm}\right)=0$ and $q\left(e^{+}, e^{-}\right)=1$. The orthogonal Lie algebra $\mathfrak{o}\left(S_{2}, q\right)$ is spanned by $\phi=\sigma_{e^{-}, e^{+}}$, which satisfies $\phi\left(e^{ \pm}\right)= \pm e^{ \pm}$, and the triality Lie algebra is

$$
\mathfrak{t r i}\left(S_{2}, \bullet, q\right)=\{(\alpha \phi, \beta \phi, \gamma \phi): \alpha, \beta, \gamma \in F, \alpha+\beta+\gamma=0\} .
$$

Besides,

$$
\begin{aligned}
t_{e^{-}, e^{+}} & =\left(\sigma_{e^{-}, e^{+}}, \frac{1}{2} q\left(e^{-}, e^{+}\right) i d-r_{e^{-}} l_{e^{+}}, \frac{1}{2} q\left(e^{-}, e^{+}\right) i d-l_{e^{-} r_{e^{+}}}\right) \\
& =\left(\phi,-\frac{1}{2} \phi,-\frac{1}{2} \phi\right) .
\end{aligned}
$$

Let $\mathfrak{t}_{2}=\left\{\left(\alpha_{0}, \alpha_{1}, \alpha_{2}\right) \in F^{3}: \alpha_{0}+\alpha_{1}+\alpha_{2}=0\right\}$, which is a two-dimensional abelian Lie algebra. For any $\sigma \in \mathcal{S}_{\mathfrak{f}_{4}} \backslash\{\emptyset,\{1,2,3,4\}\}$, consider the element $a_{\sigma} \in \mathfrak{t}_{2}$ given by $a_{\sigma}=a_{\bar{\sigma}}$, where $\bar{\sigma}=\{1,2,3,4\} \backslash \sigma$, and

$$
a_{\{1,2\}}=\left(1,-\frac{1}{2},-\frac{1}{2}\right), \quad a_{\{2,3\}}=\left(-\frac{1}{2}, 1,-\frac{1}{2}\right), \quad a_{\{1,3\}}=\left(-\frac{1}{2},-\frac{1}{2}, 1\right) .
$$

Consider too the $\sigma$-action of $\mathfrak{t}_{2}$ on $E=S_{2}=F e^{+}+F e^{-}$defined by

$$
\left(\alpha_{0}, \alpha_{1}, \alpha_{2}\right) \cdot e^{ \pm}= \pm \alpha_{i} e^{ \pm},
$$

where $i=0$ for $\sigma=\{1,2\}$ or $\{3,4\}, i=1$ for $\sigma=\{2,3\}$ or $\{1,4\}$, and $i=2$ for $\sigma=\{1,3\}$ or $\{2,4\}$.

Then, the split Lie algebra $E_{6}$ is realized as $\mathfrak{g}\left(S_{8}, S_{2}\right)$ which, according to (1.6), is described as:

$$
\mathfrak{e}_{6}=\oplus_{\sigma \in \mathcal{S}_{\mathfrak{f}_{4}}} \tilde{V}(\sigma)
$$

where

$$
\left\{\begin{array}{l}
\tilde{V}(\emptyset)=\mathfrak{s p}(V)^{4} \oplus \mathfrak{t}_{2} \\
\tilde{V}(\{1,2,3,4\})=V(\{1,2,3,4\})\left(=V_{1} \otimes V_{2} \otimes V_{3} \otimes V_{4}\right) \\
\tilde{V}(\sigma)=V(\sigma) \otimes E \quad \text { for any } \sigma \in \mathcal{S}_{\mathfrak{f}_{4}}, \sigma \neq \emptyset,\{1,2,3,4\} .
\end{array}\right.
$$

The vector space $\tilde{V}(\sigma)$ is a module for $\mathfrak{s p}(V)^{4} \oplus \mathfrak{t}_{2}$ by means of the natural action of $\mathfrak{s p}(V)^{4}$ on $V(\sigma)$ and the $\sigma$-action of $\mathfrak{t}_{2}$ on $E$ if $\sigma \in \mathcal{S}_{\mathfrak{f}_{4}} \backslash\{\emptyset,\{1,2,3,4\}\}$. 
Finally, take the bilinear maps $\varphi_{\sigma, \tau}$ defined in (2.17) and define the new bilinear maps

$$
\tilde{\varphi}_{\sigma, \tau}: \tilde{V}(\sigma) \times \tilde{V}(\tau) \rightarrow \tilde{V}(\sigma+\tau)
$$

for $\sigma, \tau \in \mathcal{S}_{\mathfrak{f}_{4}}$, as follows:

- $\tilde{\varphi} \emptyset, \emptyset$ is the Lie bracket in the direct $\operatorname{sum} \mathfrak{s p}(V)^{4} \oplus \mathfrak{t}_{2}$.

- For any $\emptyset \neq \sigma \in \mathcal{S}_{\mathfrak{f}_{4}}, \tilde{\varphi}_{\emptyset, \sigma}=-\tilde{\varphi}_{\sigma, \emptyset}$ is given by the action of $\mathfrak{s p}(V)^{4} \oplus \mathfrak{t}_{2}$ on $\tilde{V}(\sigma)$. $\left(\mathfrak{s p}(V)^{4}\right.$ acts on $V(\sigma)$ and, if present, $\mathfrak{t}_{2}$ on $E$ by means of the $\sigma$-action.)

- $\tilde{\varphi}_{\{1,2,3,4\},\{1,2,3,4\}}=\varphi_{\{1,2,3,4\},\{1,2,3,4\}}$.

- For any $\sigma \in \mathcal{S}_{\mathfrak{f}_{4}} \backslash\{\emptyset,\{1,2,3,4\}\}$,

$$
\begin{aligned}
\tilde{\varphi}_{\{1,2,3,4\}, \sigma}\left(x_{\{1,2,3,4\}}, y_{\sigma} \otimes e\right) & =\varphi_{\{1,2,3,4\}, \sigma}\left(x_{\{1,2,3,4\}}, y_{\sigma}\right) \otimes e \\
& =\tilde{\varphi}_{\sigma,\{1,2,3,4\}}\left(y_{\sigma} \otimes e, x_{\{1,2,3,4\}}\right)
\end{aligned}
$$

for any $x_{\{1,2,3,4\}} \in V(\{1,2,3,4\})=\tilde{V}(\{1,2,3,4\}), y_{\sigma} \in V(\sigma)$ and $e \in E$.

- For any $\sigma \in \mathcal{S}_{\mathfrak{f}_{4}} \backslash\{\emptyset,\{1,2,3,4\}\}$, so $\sigma=\{i, j\}$ for some $i, j \in$ $\{1,2,3,4\}$, and any $v_{i}, w_{i} \in V_{i}, v_{j}, w_{j} \in V_{j}$, and $\nu, \nu^{\prime} \in\{+,-\}$ :

$$
\begin{aligned}
& \tilde{\varphi}_{\sigma, \sigma}\left(v_{i} \otimes v_{j} \otimes e^{\nu}, w_{i} \otimes w_{j} \otimes e^{\nu^{\prime}}\right) \\
& \quad=\delta_{\nu,-\nu^{\prime}}\left(\varphi_{\sigma, \sigma}\left(v_{i} \otimes v_{j}, w_{i} \otimes w_{j}\right)+\nu\left\langle v_{i} \mid w_{i}\right\rangle\left\langle v_{j} \mid w_{j}\right\rangle a_{\sigma}\right),
\end{aligned}
$$

which belongs to $\mathfrak{s p}(V)^{4} \oplus \mathfrak{t}_{2}=\tilde{V}(\emptyset)$, where $\delta$ is the Kronecker delta; while, with $\bar{\sigma}=\{1,2,3,4\} \backslash \sigma$ as before,

$$
\tilde{\varphi}_{\sigma, \bar{\sigma}}\left(x_{\sigma} \otimes e^{\nu}, y_{\bar{\sigma}} \otimes e^{\nu^{\prime}}\right)=\delta_{\nu,-\nu^{\prime}} \varphi_{\sigma, \bar{\sigma}}\left(x_{\sigma}, y_{\bar{\sigma}}\right) \in \tilde{V}(\{1,2,3,4\}),
$$

for any $x_{\sigma} \in V(\sigma), y_{\bar{\sigma}} \in V(\bar{\sigma})$.

- Finally, for any $\sigma, \tau \in \mathcal{S}_{\mathfrak{f}_{4}} \backslash\{\emptyset,\{1,2,3,4\}\}$ with $\tau \neq \sigma, \bar{\sigma}$,

$$
\tilde{\varphi}_{\sigma, \tau}\left(x_{\sigma} \otimes e^{\nu}, y_{\tau} \otimes e^{\nu^{\prime}}\right)=\delta_{\nu, \nu^{\prime}} \varphi_{\sigma, \tau}\left(x_{\sigma}, y_{\tau}\right) \otimes e^{-\nu}
$$

for any $x_{\sigma} \in V(\sigma), y_{\tau} \in V(\tau)$ and $\nu, \nu^{\prime} \in\{+,-\}$.

Again, the multiplication in $\mathfrak{e}_{6}=\mathfrak{g}\left(S_{8}, S_{2}\right)$ is given by

$$
\left[x_{\sigma}, y_{\tau}\right]=\epsilon_{\mathfrak{e}_{6}}(\sigma, \tau) \tilde{\varphi}\left(x_{\sigma}, y_{\tau}\right),
$$

for any $\sigma, \tau \in \mathcal{S}_{\mathfrak{f}_{4}}, x_{\sigma} \in \tilde{V}(\sigma), y_{\tau} \in \tilde{V}(\tau)$, where

$$
\epsilon_{\mathfrak{e}_{6}}(\sigma, \tau)= \begin{cases}\frac{1}{2} \epsilon_{\mathfrak{f}_{4}}(\sigma, \tau) & \text { if } \sigma, \tau \in \mathcal{S}_{\mathfrak{f}_{4}} \backslash\{\emptyset,\{1,2,3,4\}\}, \tau=\sigma \text { or } \tau=\bar{\sigma} \\ \epsilon_{\mathfrak{f}_{4}}(\sigma, \tau) & \text { otherwise. }\end{cases}
$$


Remark 3.24 Note that in characteristic $3, a_{\{1,2\}}=a_{\{2,3\}}=a_{\{1,3\}}=$ $(1,1,1)$, and hence $\hat{\mathfrak{e}}_{6}=\left[\mathfrak{e}_{6}, \mathfrak{e}_{6}\right]=\oplus_{\sigma \in \mathcal{S}_{\mathfrak{f}_{4}}} \hat{V}(\sigma)$, with $\hat{V}(\sigma)=\tilde{V}(\sigma)$ for any $\sigma \neq \emptyset$, but with $\hat{V}(\emptyset)=\mathfrak{s p}(V)^{4} \oplus \hat{\mathfrak{t}}_{2}$, where $\hat{\mathfrak{t}}_{2}=F a_{\{1,2\}}$. Actually, the Lie algebra obtained by taking the $\mathbb{Z}$-span of a Chevalley basis of the complex simple Lie algebra of type $E_{6}$, and then tensoring with a field of characteristic three, is no longer simple, but has a one dimensional center (see, for instance, [9], or [33, §3]). Modulo this center, one has a simple Lie algebra of dimension 77 , which is isomorphic to $\hat{\mathfrak{e}}_{6}$, and $\mathfrak{e}_{6}$ is isomorphic to the Lie algebra of derivations $\operatorname{Der}\left(\hat{\mathfrak{e}}_{6}\right)$.

Also, the split Lie algebra $A_{5}$ is realized as $\mathfrak{a}_{5}=\mathfrak{g}\left(S_{4}, S_{2}\right)$, a subalgebra of $\mathfrak{e}_{6}=\mathfrak{g}\left(S_{8}, S_{2}\right)$. Thus, by restriction, one gets

$$
\mathfrak{a}_{5}=\oplus_{\sigma \in \mathcal{S}_{\mathfrak{c}_{3}}} \hat{V}(\sigma),
$$

where $\hat{V}(\emptyset)=\mathfrak{s p}(V)^{3} \oplus \mathfrak{t}_{2}$ and $\hat{V}(\sigma)=\tilde{V}(\sigma)$ for any $\emptyset \neq \sigma \in \mathcal{S}_{\mathfrak{c}_{3}}$, and the multiplication is given by

$$
\left[x_{\sigma}, y_{\tau}\right]=\epsilon_{\mathfrak{a}_{5}}(\sigma, \tau) \tilde{\varphi}\left(x_{\sigma}, y_{\tau}\right),
$$

for any $\sigma, \tau \in \mathcal{S}_{\mathfrak{c}_{3}}, x_{\sigma} \in \hat{V}(\sigma), y_{\tau} \in \hat{V}(\tau)$, with

$$
\epsilon_{\mathfrak{a}_{5}}(\sigma, \tau)= \begin{cases}\frac{1}{2} \epsilon_{\mathfrak{c}_{3}}(\sigma, \tau) & \text { if } \sigma=\tau \neq \emptyset \\ \epsilon_{\mathfrak{c}_{3}}(\sigma, \tau) & \text { otherwise }\end{cases}
$$

Also here, if the characteristic is $3, \mathfrak{a}_{5}$ is no longer simple, since it contains the simple codimension one ideal $\hat{\mathfrak{a}}_{5}=\left[\mathfrak{a}_{5}, \mathfrak{a}_{5}\right]$, which is isomorphic to the projective special linear algebra $\mathfrak{p s l}_{6}(F)$. It can be checked that $\mathfrak{a}_{5}$ is isomorphic to the projective general linear algebra $\mathfrak{p g l}_{6}(F)$.

Remark 3.27 In concluding this section, let us remark that the models of the exceptional simple Lie algebras obtained provide, in particular, very easy descriptions of the exceptional root systems, different from the description in [7]. Thus, for instance, for $E_{8}$, take a symplectic basis $\left\{v_{i}, w_{i}\right\}$ of $V_{i}(i=1, \ldots, 8)$ (that $\left.i s,\left\langle v_{i} \mid w_{i}\right\rangle=1\right)$. Then the vector space $\mathfrak{h}_{8}=$ $\operatorname{span}\left\{\gamma_{v_{i}, w_{i}}: i=1, \ldots, 8\right\}\left(\subseteq V(\emptyset)=\bigoplus_{i=1}^{8} \mathfrak{s p}\left(V_{i}\right)\right)$ is a Cartan subalgebra of $\mathfrak{e}_{8}$. Since $\gamma_{v_{i}, w_{i}}\left(v_{i}\right)=-v_{i}, \gamma_{v_{i}, w_{i}}\left(w_{i}\right)=w_{i}$, and hence also $\left[\gamma_{v_{i}, w_{i}}, \gamma_{v_{i}, v_{i}}\right]=$ $-2 \gamma_{v_{i}, v_{i}}$ and $\left[\gamma_{v_{i}, w_{i}}, \gamma_{w_{i}, w_{i}}\right]=2 \gamma_{w_{i}, w_{i}}$, let $\varepsilon_{i}: \mathfrak{h}_{8} \rightarrow F$ be the linear map given by $\varepsilon_{i}\left(\gamma_{v_{j}, w_{j}}\right)=\delta_{i j}$ for any $1 \leq i, j \leq 8$. The description of $\mathfrak{e}_{8}$ in 3.15 shows that the corresponding root system is

$$
\begin{aligned}
\Phi=\left\{ \pm 2 \varepsilon_{i}: i=\right. & 1, \ldots, 8\} \\
& \cup\left\{ \pm \varepsilon_{i_{1}} \pm \varepsilon_{i_{2}} \pm \varepsilon_{i_{3}} \pm \varepsilon_{i_{4}}: \sigma=\left\{i_{1}, i_{2}, i_{3}, i_{4}\right\} \in \mathcal{S}_{\mathfrak{e}_{8}} \backslash\{\emptyset\}\right\} .
\end{aligned}
$$


Alternatively (see Remark 3.20), if the points of the affine space $\mathbb{Z}_{2}^{3}$ are numbered from 1 to 8 , then

$$
\begin{aligned}
\Phi= & \left\{ \pm 2 \varepsilon_{i}: i=1, \ldots, 8\right\} \\
& \cup\left\{ \pm \varepsilon_{i_{1}} \pm \varepsilon_{i_{2}} \pm \varepsilon_{i_{3}} \pm \varepsilon_{i_{4}}: \sigma=\left\{i_{1}, i_{2}, i_{3}, i_{4}\right\} \text { is an affine plane in } \mathbb{Z}_{2}^{3}\right\} .
\end{aligned}
$$

Using the lexicographic order with $0<\varepsilon_{1}<\varepsilon_{2}<\cdots<\varepsilon_{8}$, the simple system of roots is $\Delta=\left\{\alpha_{1}, \ldots, \alpha_{8}\right\}$ (numbering as in [7]) with $\alpha_{1}=-\varepsilon_{1}-\varepsilon_{4}-\varepsilon_{6}+\varepsilon_{7}$, $\alpha_{2}=2 \varepsilon_{2}, \alpha_{3}=2 \varepsilon_{1}, \alpha_{4}=-\varepsilon_{1}-\varepsilon_{2}-\varepsilon_{3}+\varepsilon_{4}, \alpha_{5}=2 \varepsilon_{3}, \alpha_{6}=-\varepsilon_{3}-\varepsilon_{4}-\varepsilon_{5}+\varepsilon_{6}$, $\alpha_{7}=2 \varepsilon_{5}$ and $\alpha_{8}=-\varepsilon_{5}-\varepsilon_{6}-\varepsilon_{7}+\varepsilon_{8}$. (For $\mathfrak{e}_{7}$ it is enough to suppress here $\varepsilon_{8}$ and $\alpha_{8}$ above or, alternatively, the points of the Fano plane can be numbered from 1 to 7 , and the root system of $\mathfrak{e}_{7}$ becomes $\left\{ \pm 2 \varepsilon_{i}: i=1, \ldots, 7\right\} \cup\left\{ \pm \varepsilon_{i_{1}} \pm\right.$ $\varepsilon_{i_{2}} \pm \varepsilon_{i_{3}} \pm \varepsilon_{i_{4}}: \sigma=\left\{i_{1}, i_{2}, i_{3}, i_{4}\right\}$ is a quadrilateral in $\left.\mathbb{Z}_{2} \mathbb{P}^{2}\right\}$.)

\section{Freudenthal triple systems}

Freudenthal $[19,20]$ obtained the exceptional simple Lie algebras in terms of some triple systems, later called Freudenthal triple systems. Actually, if $T$ is such a system, the direct sum of two copies of $T$ becomes a Lie triple system, and hence the odd part of a $\mathbb{Z}_{2}$-graded Lie algebra. In this section, the exceptional split simple Freudenthal triple systems will be recovered from the models given in the previous section of the exceptional simple Lie algebras. For our purposes, the approach given by Yamaguti and Asano [32] of Freudenthal's construction is more suitable.

Let us first recall some definitions and results.

Let $T$ be a vector space endowed with a nonzero alternating bilinear form (.|.) $: T \times T \rightarrow F$, and a triple product $T \times T \times T \rightarrow T:(x, y, z) \mapsto[x y z]$. Then $(T,[\ldots],(. \mid)$.$) is said to be a symplectic triple system (see [32]) if it$ satisfies the following identitities:

$$
\begin{aligned}
& {[x y z]=[y x z]} \\
& {[x y z]-[x z y]=(x \mid z) y-(x \mid y) z+2(y \mid z) x} \\
& {[x y[u v w]]=[[x y u] v w]+[u[x y v] w]+[u v[x y w]]} \\
& ([x y u] \mid v)+(u \mid[x y v])=0
\end{aligned}
$$

for any elements $x, y, z, u, v, w \in T$.

Note that $(4.1 \mathrm{~b})$ can be written as

$$
[x y z]-[x z y]=\psi_{x, y}(z)-\psi_{x, z}(y)
$$

with $\psi_{x, y}(z)=(x \mid z) y+(y \mid z) x$. (The maps $\psi_{x, y}$ span the symplectic Lie algebra $\mathfrak{s p}(T)$.) 
Also, (4.1c) is equivalent to $d_{x, y}=[x y$. $]$ being a derivation of the triple system. Let inder $(T)$ be the linear span of $\left\{d_{x, y}: x, y \in T\right\}$, which is a Lie subalgebra of $\operatorname{End}(T)$. For any $x, y, z, a, b \in T$, by (4.1b) and (4.1c),

$$
\begin{aligned}
0= & {[x y([z a b]-[z b a])]-(z \mid b)[x y a]+(z \mid a)[x y b]-2(a \mid b)[x y z] } \\
= & ([[x y z] a b]-[[x y z] b a])+([z[x y a] b]-[z b[x y a]]) \\
& +([z a[x y b]]-[z[x y b] a])-(z \mid b)[x y a]+(z \mid a)[x y a]-2(a \mid b)[x y z] \\
= & (([x y z] \mid b)+(z \mid[x y b])) a-(([x y z] \mid a)+(z \mid[x y a])) b \\
& +2(([x y a] \mid b)+(a \mid[x y b])) z .
\end{aligned}
$$

Hence, if $\operatorname{dim} T \geq 3,(4.1 \mathrm{~d})$ (that is, $\operatorname{inder}(T) \subseteq \mathfrak{s p}(T)$ ) follows from (4.1b) and (4.1c). Also, with $a=z$ (4.3) gives

$$
3(([x y a] \mid b)+(a \mid[x y b]))=0,
$$

so the same applies if the characteristic of $F$ is $\neq 3$. This was already noted, over fields of characteristic 0 , in [32]. However, this is no longer true if the characteristic of $F$ is 3 and $\operatorname{dim} T=2$. First, notice that in this case, the right hand side of (4.1b) becomes

$$
(x \mid z) y+(y \mid x) z+(z \mid y) x
$$

which is 0 , since it is skew symmetric on its $\operatorname{arguments}$ and $\operatorname{dim} T=2$. Hence (4.1a) and (4.1b) merely say that [...] is symmetric on its arguments.

As a counterexample in characteristic 3 , take $T=F a+F b$ with $(a \mid b)=1$ and $[\ldots]$ determined by $[a a a]=[b b b]=[a b b]=0$ and $[a a b]=b$. One checks easily that (4.1c) is satisfied, but $d_{a, a} \notin \mathfrak{s p}(T)$, so (4.1d) is not satisfied.

Symplectic triple systems are strongly related to a particular kind of $\mathbb{Z}_{2}$-graded Lie algebras:

Theorem 4.4 Let $(T,[\ldots],(. \mid)$.$) be a symplectic triple system and let (V,\langle. \mid\rangle$. be a two dimensional vector space endowed with a nonzero alternating bilinear form. Define the $\mathbb{Z}_{2}$-graded algebra $\mathfrak{g}=\mathfrak{g}_{\overline{0}} \oplus \mathfrak{g}_{\overline{1}}$ with

$$
\left\{\begin{array}{l}
\mathfrak{g}_{\overline{0}}=\operatorname{inder}(T) \oplus \mathfrak{s p}(V) \quad \text { (direct sum of ideals) } \\
\mathfrak{g}_{\overline{1}}=T \otimes V
\end{array}\right.
$$

and anticommutative multiplication given by:

- $\mathfrak{g}_{\overline{0}}$ is a Lie subalgebra of $\mathfrak{g}$,

- $\mathfrak{g}_{\overline{0}}$ acts naturally on $\mathfrak{g}_{\overline{1}}$; that is

$$
[d, x \otimes v]=d(x) \otimes v, \quad[s, x \otimes v]=x \otimes s(v),
$$

for any $d \in \operatorname{inder}(T), s \in \mathfrak{s p}(V), x \in T$, and $v \in V$. 
- For any $x, y \in T$ and $u, v \in V$ :

$$
[x \otimes u, y \otimes v]=\langle u \mid v\rangle d_{x, y}+(x \mid y) \gamma_{u, v}
$$

where $\gamma_{u, v}=\langle u \mid\rangle v+.\langle v \mid\rangle$.$u .$

Then $\mathfrak{g}$ is a Lie algebra.

Conversely, given a $\mathbb{Z}_{2}$-graded Lie algebra $\mathfrak{g}=\mathfrak{g}_{\overline{0}} \oplus \mathfrak{g}_{\overline{1}}$ with

$$
\begin{cases}\mathfrak{g}_{\overline{0}}=\mathfrak{s} \oplus \mathfrak{s p}(V) & \text { (direct sum of ideals), } \\ \mathfrak{g}_{\overline{1}}=T \otimes V & \text { (as a module for } \left.\mathfrak{g}_{\overline{0}}\right),\end{cases}
$$

where $T$ is a module for $\mathfrak{s}$, by $\mathfrak{g}_{0}$-invariance of the Lie bracket, equation (4.5) is satisfied for an alternating bilinear form (.|.) $: T \times T \rightarrow F$ and a symmetric bilinear map $d_{., .}: T \times T \rightarrow \mathfrak{s}$. Then, if (.|.) is not 0 and a triple product on $T$ is defined by means of $[x y z]=d_{x, y}(z),(T,[\ldots],(. \mid)$.$) is a symplectic$ triple system.

Proof. This is a reformulation and extension of the results in [32, p. 256], suitable for our purposes. First, since $[x y.] \in \mathfrak{s p}(T)$ for any $x, y \in T$, a straightforward computation shows that the Jacobi identity holds in the algebra $\mathfrak{g}$.

Conversely, if $\mathfrak{g}=(\mathfrak{s} \oplus \mathfrak{s p}(V)) \oplus(T \otimes V)$ is a Lie algebra as above, (4.1a) follows by the symmetry of $d_{x, y}$. Also, the Jacobi identity with an element of $\mathfrak{s}$ and two basic tensors in $T \otimes V$ shows that (.|.) and the map $(x, y) \mapsto d_{x, y}$ are $\mathfrak{s - i n v a r i a n t}$ and, in particular, one gets (4.1c). Finally, the Jacobi identity $J(x \otimes w, y \otimes v, z \otimes v)=0$ with $x, y, z \in T$ and $\langle v \mid w\rangle=1$ gives (4.1b).

Note that the Lie algebra $\mathfrak{g}$ in Theorem 4.4 is graded by the root system $\mathrm{BC}_{1}$ with grading subalgebra of type $\mathrm{C}_{1}$ (see $[6]$ ).

If $\{v, w\}$ is a symplectic basis of $V$ (notation as in the previous Theorem), so $\langle v \mid w\rangle=1$, then with $h=-\gamma_{v, w}, e=\frac{1}{2} \gamma_{v, v}$ and $f=-\frac{1}{2} \gamma_{w, w},\{h, e, f\}$ is a canonical basis of $\mathfrak{s p}(V) \cong \mathfrak{s l}_{2}([h, e]=2 e,[h, f]=-2 f,[e, f]=h)$ with $h(v)=v, h(w)=-w$. Hence the Lie algebra $\mathfrak{g}$ above is 5 -graded

$$
\mathfrak{g}=\mathfrak{g}_{-2} \oplus \mathfrak{g}_{-1} \oplus \mathfrak{g}_{0} \oplus \mathfrak{g}_{1} \oplus \mathfrak{g}_{2}
$$

where $\mathfrak{g}_{i}=\{x \in \mathfrak{g}:[h, x]=i x\}$ for any $i$. Here $\mathfrak{g}_{2}=F e, \mathfrak{g}_{-2}=F f$, $\mathfrak{g}_{1}=T \otimes v, \mathfrak{g}_{-1}=T \otimes w$ and $\mathfrak{g}_{0}=\mathfrak{s} \oplus F h$.

Now, let $T$ be a vector space endowed with a nonzero alternating bilinear form (.|.) $: T \times T \rightarrow F$, and a triple product $T \times T \times T \rightarrow T,(x, y, z) \mapsto x y z$. 
Then $(T, x y z,(. \mid)$.$) is said to be a Freudenthal triple system (see [29,18,8]$ ) if it satisfies:

(4.6a) $x y z$ is symmetric in its arguments,

(4.6b) (x|yzt) is symmetric in its arguments,

(4.6c) $(x y y) x z+(y x x) y z+(x y y \mid z) x+(y x x \mid z) y+(x \mid z) x y y+(y \mid z) y x x=0$

for any $x, y, z, t \in T$.

Note that the bilinear form (.|.) is not assumed to be nondegenerate.

Theorem 4.7 Let (.|.) be an alternating bilinear form on the vector space $T$ and let $x y z$ and $[x y z]$ be two triple products on $T$ related by $x y z=[x y z]-$ $\psi_{x, y}(z)$ for any $x, y, z \in T$. Then $(T, x y z,(. \mid)$.$) is a Freudenthal triple system$ if and only if $(T,[x y z],(. \mid)$,$) is a symplectic triple system.$

Proof. First assume that $(T,[\ldots],(. \mid)$.$) is a symplectic triple system and$ define $x y z=[x y z]-\psi_{x, y}(z)$ for any $x, y, z \in T$. Then $x y z$ is symmetric in $x$ and $y$ since so are $[x y z]$ and $\psi_{x, y}$, while (4.2) implies that $x y z$ is symmetric in $y$ and $z$, thus proving (4.6a). Also, (x|yzt) is symmetric in $y, z$ and $t$ and, since both [yz.] and $\psi_{y, z}$ belong to $\mathfrak{s p}(T)$, it follows that $(x \mid y z t)$ is symmetric too in $x$ and $t$, proving (4.6b). Finally, with $d_{x, y}=[x y$.] for any $x, y \in T$,

$$
\begin{aligned}
& d_{x, x y y}=d_{x, d_{x, y}(y)}-d_{x, \psi_{x, y}(y)}=d_{x, d_{x, y}(y)}-(x \mid y) d_{x, y} \\
& d_{y, y x x}=d_{y, d_{y, x}(x)}-d_{y, \psi_{y, x}(x)}=d_{y, d_{x, y}(x)}+(x \mid y) d_{x, y}
\end{aligned}
$$

so by $(4.1 c)$

$$
d_{x, x y y}+d_{y, y x x}=d_{x, d_{x, y}(y)}+d_{d_{x, y}(x), y}=\left[d_{x, y}, d_{x, y}\right]=0 .
$$

Thus,

$$
d_{x, x y y}+d_{y, y x x}=0
$$

for any $x, y \in T$, and this is equivalent to (4.6c).

Conversely, assume that $(T, x y z,(. \mid)$.$) is a Freudenthal triple system.$ Then (4.1a) and (4.1b) follow from (4.6a), while (4.6a) and (4.6b) imply (4.1d). It is enough then to prove that $d_{x, x}=[x x]:. y \mapsto x x y+\psi_{x, x}(y)$ is a derivation of $(T,[\ldots])$ for any $x \in T$, and since $d_{T, T} \subseteq \mathfrak{s p}(T)$, it suffices to show that $d_{x, x}$ is a derivation of the Freudenthal triple system or, equivalently, that

$$
d_{x, x}(y y z)=2\left(d_{x, x}(y)\right) y z+y y\left(d_{x, x}(z)\right)
$$

for any $x, y, z \in T$. 
Linearizing (4.6c) in $y$, then taking $z=y$ and using (4.6a) and (4.6b) one obtains

$$
\begin{aligned}
2(x y t) x y+(y x x) y t+(x x t) y y+2(x y t \mid y) x \\
+(y x x \mid y) t+(t x x \mid y) y+2(x \mid y) x y t+(t \mid y) y x x=0
\end{aligned}
$$

Now, interchange $x$ and $y$ in (4.9) and subtract the result from (4.9) to get

$$
\begin{aligned}
(x x y) y t-(y y x) x t+y y(x x t)-x x(y y t)-(x \mid y y t) x+(y \mid x x t) y & \\
+4(x \mid y) x y t+(t \mid y) y x x-(t \mid x) x y y & =0 .
\end{aligned}
$$

Adding (4.10) and (4.6c) with $z=t$ gives

$$
2(x x y) y t+y y(x x t)-x x(y y t)-2(x \mid y y t) x+4(x \mid y) x y t+2(x \mid t) x y y=0,
$$

which is equivalent to (4.8).

Remark 4.11 There are several results in the literature relating different triple systems. See, for instance, [24, Section 2], [17] or [8].

Related constructions of a 5-graded Lie algebra from Freudenthal triple systems (or equivalent ternary algebras) are given in [16, 25].

The models of the exceptional Lie algebras in Section 3 fit in the situation described in Theorem 4.4. Therefore, by Theorem 4.7, they provide models of Freudenthal triple systems.

Take for instance our model for $\mathfrak{e}_{8}$ (3.15). For any $\sigma \in \mathcal{S}_{\mathfrak{e}_{8}} \backslash \mathcal{S}_{\mathfrak{e}_{7}}, 8 \in \sigma$ and (3.15) can be rewritten as

$$
\mathfrak{e}_{8}=\left(\mathfrak{e}_{7} \oplus \mathfrak{s p}\left(V_{8}\right)\right) \bigoplus\left(\left(\bigoplus_{\sigma \in \mathcal{S}_{\mathfrak{e}_{8} \backslash \mathcal{S}_{\mathfrak{e}_{7}}}} V(\sigma \backslash\{8\})\right) \otimes V_{8}\right)
$$

and we are in the situation of Theorem 4.4. In particular,

$$
\mathcal{T}_{\mathfrak{e}_{8}, \mathfrak{e}_{7}}=\bigoplus_{\sigma \in \mathcal{S}_{\mathfrak{e}_{8}} \backslash \mathcal{S}_{\mathfrak{e}_{7}}} V(\sigma \backslash\{8\})
$$

is a simple Freudenthal triple system of dimension $2^{3} \times 7=56$, whose triple product and nondegenerate alternating bilinear form can be easily computed in terms of the invariant maps $\varphi_{\sigma, \tau}$ in (2.17). 
In the same vein,

$$
\begin{aligned}
& \mathfrak{e}_{7}=\left(\mathfrak{d}_{6} \oplus \mathfrak{s p}\left(V_{4}\right)\right) \bigoplus\left(\left(\bigoplus_{\sigma \in \mathcal{S}_{\mathfrak{e}_{7} \backslash \mathcal{S}_{\mathfrak{d}_{6}}}} V(\sigma \backslash\{4\})\right) \otimes V_{4}\right), \\
& \mathfrak{e}_{6}=\left(\mathfrak{a}_{5} \oplus \mathfrak{s p}\left(V_{4}\right)\right) \bigoplus\left(\left(\bigoplus_{\sigma \in \mathcal{S}_{\mathfrak{f}_{4} \backslash \mathcal{S}_{\mathfrak{c}_{3}}}} \tilde{V}(\sigma \backslash\{4\})\right) \otimes V_{4}\right), \\
& \left.\mathfrak{f}_{4}=\left(\mathfrak{c}_{3} \oplus \mathfrak{s p}\left(V_{4}\right)\right) \bigoplus\left(\bigoplus_{\sigma \in \mathcal{S}_{\mathfrak{f}_{4} \backslash \mathcal{S}_{\mathfrak{c}_{3}}}} V(\sigma \backslash\{4\})\right) \otimes V_{4}\right),
\end{aligned}
$$

and we obtain simple Freudenthal triple systems defined on:

$$
\begin{aligned}
& \mathcal{T}_{\mathfrak{e}_{7}, \mathfrak{d}_{6}}=\bigoplus_{\sigma \in \mathcal{S}_{\mathfrak{S}_{7} \backslash \mathcal{S}_{\mathfrak{d}_{6}}} V(\sigma \backslash\{4\}),} V(\sigma) \\
& \mathcal{T}_{\mathfrak{e}_{6}, \mathfrak{a}_{5}}=\bigoplus_{\sigma \in \mathcal{S}_{\mathfrak{f}_{4} \backslash \mathcal{S}_{\mathfrak{c}_{3}}}} \tilde{V}(\sigma \backslash\{4\}), \\
& \mathcal{T}_{\mathfrak{f}_{4}, \mathfrak{c}_{3}}=\bigoplus_{\sigma \in \mathcal{S}_{\mathfrak{f}_{4} \backslash \mathcal{S}_{\mathfrak{c}_{3}}}} V(\sigma \backslash\{4\}),
\end{aligned}
$$

of dimension $2^{3} \times 4=32,2^{3}+2^{2} \times 3=20$ and $2^{3}+2 \times 3=14$, respectively.

The classification in [29] and [8] implies that, over algebraically closed fields, these are the simple Freudenthal triple systems associated to the split simple Jordan algebras of degree 3, that is, the Freudenthal triple systems originally considered by Freudenthal.

\section{References}

[1] Albuquerque, H. And Majid, S.: Quasialgebra structure of the octonions. J. Algebra 220 (1999), no. 1, 188-224.

[2] Allison, B. N. and Faulkner, J. R.: Nonassociative coefficient algebras for Steinberg unitary Lie algebras. J. Algebra 161 (1993), no. 1, 1-19.

[3] Baez, J. C.: The octonions. Bull. Amer. Math. Soc. (N.S.) 39 (2002), no. 2, 145-205 (electronic).

[4] Barton, C. H. And Sudbery, A.: Magic squares of Lie algebras. arXiv: math. RA/0001083.

[5] Barton, C. H. And Sudbery, A.: Magic squares and matrix models of Lie algebras. Adv. Math. 180 (2003), no. 2, 596-647.

[6] Benkart, G. and Smirnov, O.: Lie algebras graded by the root system $\mathrm{BC}_{1}$. J. Lie Theory 13 (2003), no. 1, 91-132. 
[7] Bourbaki, N.: Lie groups and Lie algebras. Chapters 4-6. Elements of Mathematics (Berlin), translated from the 1968 French original by Andrew Pressley, Springer-Verlag, Berlin, 2002.

[8] Brown, G.: Freudenthal triple systems of characteristic three. Algebras Groups Geom. 1 (1984), no. 4, 399-441.

[9] Dieudonné, J.: Les algèbres de Lie simples associées aux groupes simples algèbriques sur un corps de caractéristique $p>0$. Rend. Circ. Mat. Palermo (2) 6 (1957), 198-204.

[10] Elduque, A.: Symmetric composition algebras. J. Algebra 196 (1997), no. $1,282-300$.

[11] Elduque, A.: The magic square and symmetric compositions. Rev. Mat. Iberoamericana 20 (2004), 475-491.

[12] Elduque, A.: A new look at Freudenthal's Magic Square. In Nonassociative algebra and its applications, 149-165, Lect. Notes Pure Appl. Math. 246, Chapman \& Hall/CRC, Boca Raton, FL, 2006.

[13] Elduque, A. And Myung, H. C.: Flexible composition algebras and Okubo algebras. Comm. Algebra 19 (1991), no. 4, 1197-1227.

[14] Elduque, A. And Myung, H. C.: On flexible composition algebras. Comm. Algebra 21 (1993), no. 7, 2481-2505.

[15] Elduque, A. And PÉrez, J. M.: Composition algebras with associative bilinear form. Comm. Algebra 24 (1996), no. 3, 1091-1116.

[16] Faulkner, J. R.: A construction of Lie algebras from a class of ternary algebras. Trans. Amer. Math. Soc. 155 (1971), 397-408.

[17] Faulkner, J.R. AND Ferrar, J. C.: On the structure of symplectic ternary algebras. Nederl. Akad. Wetensch. Proc. Ser. A $\mathbf{7 5}=$ Indag. Math. 34 (1972), 247-256.

[18] Ferrar, J. C.: Strictly regular elements in Freudenthal triple systems. Trans. Amer. Math. Soc. 174 (1972), 313-331.

[19] Freudenthal, H.: Beziehungen der $\mathfrak{E}_{7}$ und $\mathfrak{E}_{8}$ zur Oktavenebene. II. Nederl. Akad. Wetensch. Proc. Ser. A. 57 = Indag. Math. 16 (1954), 363368.

[20] Freudenthal, H.: Beziehungen der $\mathfrak{E}_{7}$ und $\mathfrak{E}_{8}$ zur Oktavenebene. VIII. Nederl. Akad. Wetensch. Proc. Ser. A. 62 = Indag. Math. 21 (1959), 447465.

[21] Grishkov, A. N.: The automorphisms group of the multiplicative Cartan decomposition of Lie algebra $E_{8}$. Internat. J. Algebra Comput. 11 (2001), no. $6,737-752$.

[22] Grishkov, A. N.: Lie algebras with triality. J. Algebra 266 (2003), no. 2, 698-722.

[23] Jacobson, N.: Composition algebras and their automorphisms. Rend. Circ. Mat. Palermo (2) 7 (1958), 55-80. 
[24] Kamiya, N.: A structure theory of Freudenthal-Kantor triple systems. III. Mem. Fac. Sci. Shimane Univ. 23 (1989), 33-51.

[25] Kantor, I. L. And Skopec, I. M.: Freudenthal trilinear systems. Trudy Sem. Vektor. Tenzor. Anal. 18 (1978), 250-263.

[26] Knus, M-A., Merkurjev, A., Rost, M. and Tignol, J-P.: The book of involutions. American Mathematical Society Colloquium Publications 44. American Mathematical Society, Providence, RI, 1998.

[27] Landsberg, J. M. and Manivel, L.: Triality, exceptional Lie algebras and Deligne dimension formulas. Adv. Math. 171 (2002), no. 1, 59-85.

[28] Landsberg, J. M. And Manivel, L.: Representation theory and projective geometry. In Algebraic transformation groups and algebraic varieties, 71-122. Encyclopaedia Math. Sci. 132. Springer, Berlin, 2004.

[29] Meyberg, K.: Eine Theorie der Freudenthalschen Tripelsysteme. I, II. Nederl. Akad. Wetensch. Proc. Ser. A 71=Indag. Math. 30 (1968), 162174, 175-190.

[30] Onishchik, A. L. And Vinberg, E. B.: Lie groups and Lie algebras, III. Encyclopaedia of Mathematical Sciences 41. Springer-Verlag, Berlin, 1994.

[31] Tits, J.: Algèbres alternatives, algèbres de Jordan et algèbres de Lie exceptionnelles. I. Construction. Nederl. Akad. Wetensch. Proc. Ser. A $69=$ Indag. Math. 28 (1966), 223-237.

[32] Yamaguti, K. And Asano, H.: On the Freudenthal's construction of exceptional Lie algebras. Proc. Japan Acad. 51 (1975), no. 4, 253-258.

[33] VeǏsfeǏLeR, B. Ju. AND KaC, V. G.: Exponentials in Lie algebras of characteristic p. Izv. Akad. Nauk SSSR Ser. Mat. 35 (1971), 762-788.

Recibido: 17 de noviembre de 2004

Revisado: 28 de abril de 2005

Alberto Elduque

Departamento de Matemáticas

Universidad de Zaragoza

50009-Zaragoza, Spain

elduque@unizar.es

Supported by the Spanish Ministerio de Ciencia y Tecnología and FEDER (BFM 20013239-C03-03), by the Ministerio de Educación y Ciencia and FEDER (MTM 2004-08115C04-02), and by the Diputación General de Aragón (Grupo de Investigación de Álgebra). 